\title{
The Roquette category of finite $p$-groups
}

\author{
Serge Bouc
}

\begin{abstract}
Let $p$ be a prime number. This paper introduces the Roquette category $\mathcal{R}_{p}$ of finite $p$-groups, which is an additive tensor category containing all finite $p$-groups among its objects. In $\mathcal{R}_{p}$, every finite $p$-group $P$ admits a canonical direct summand $\partial P$, called the edge of $P$. Moreover $P$ splits uniquely as a direct sum of edges of Roquette p-groups, and the tensor structure of $\mathcal{R}_{p}$ can be described in terms of such edges.

The main motivation for considering this category is that the additive functors from $\mathcal{R}_{p}$ to abelian groups are exactly the rational p-biset functors. This yields in particular very efficient ways of computing such functors on arbitrary $p$-groups : this applies to the representation functors $R_{K}$, where $K$ is any field of characteristic 0 , but also to the functor of units of Burnside rings, or to the torsion part of the Dade group.
\end{abstract}

AMS Subject classification : 18B99, 19A22, 20C99, 20J15.

Keywords : p-group, Roquette, rational, biset, genetic.

\section{Introduction}

Let $p$ be a prime number. This article introduces the Roquette category $\mathcal{R}_{p}$ of finite $p$-groups, which is an additive tensor category with the following properties :

- Every finite $p$-group can be viewed as an object of $\mathcal{R}_{p}$. The tensor product of two finite $p$-groups $P$ and $Q$ in $\mathcal{R}_{p}$ is the direct product $P \times Q$.

- In $\mathcal{R}_{p}$, any finite $p$-group has a direct summand $\partial P$, called the edge of $P$, such that

$$
P \cong \underset{N \unlhd P}{\oplus} \partial(P / N)
$$

Moreover, if the center of $P$ is not cyclic, then $\partial P=0$.

- In $\mathcal{R}_{p}$, every finite $p$-group $P$ decomposes as a direct sum

$$
P \cong \underset{R \in \mathcal{S}}{\oplus} \partial R
$$

where $\mathcal{S}$ is a finite sequence of Roquette groups, i.e. of $p$-groups of normal $p$-rank 1 , and such a decomposition is essentially unique. Given the group $P$, such a decomposition can be obtained explicitly from the knowledge of a genetic basis of $P$ (Theorem 3.11 and Proposition 5.14). 
- The tensor product $\partial P \times \partial Q$ of the edges of two Roquette $p$-groups $P$ and $Q$ is isomorphic to a direct sum of a certain number $\nu_{P, Q}$ of copies of the edge $\partial(P \diamond Q)$ of another Roquette group (where both $\nu_{P, Q}$ and $P \diamond Q$ are known explicitly - see Theorem 4.20 and Corollary 4.24).

- The additive functors from $\mathcal{R}_{p}$ to the category of abelian groups are exactly the rational p-biset functors introduced in [4].

The latter is the main motivation for considering this category : any structural result on $\mathcal{R}_{p}$ will provide for free some information on such rational functors for $p$-groups, e.g. the representation functors $R_{K}$, where $K$ is a field of characteristic 0 (see [2], 3], and L. Barker's article [1]), the functor of units of Burnside rings ([6]), or the torsion part of the Dade group ([5]).

In particular, the above results on $\mathcal{R}_{p}$ yield isomorphisms describing the structure of some $p$-groups as objects of this category, and this is enough to compute the evaluations of rational $p$-biset functors. For example

$$
\left(D_{8}\right)^{n} \cong \mathbf{1} \oplus\left(5^{n}-1\right) \cdot \partial C_{2}
$$

in $\mathcal{R}_{2}$ (Equation 5.25). More generally, Proposition 5.41 gives a formula for $\left(D_{2^{m}}\right)^{n}$. A straightforward consequence, applying the functor $R_{\mathbb{Q}}$, is the following

Example: For any $n \in N$, the group $\left(D_{8}\right)^{n}$ has $5^{n}$ conjugacy classes of cyclic subgroups.

Another important by-product of the above result giving the tensor structure of $\mathcal{R}_{p}$ is the explicit description of a genetic basis of a direct product $P \times Q$, in terms of a genetic basis of $P$ and a genetic basis of $Q$ (Theorem 5.20). This allows in particular for a quick computation of the torsion part of the Dade group of some p-groups, e.g. (Theorem 5.36, Assertion 1 and Assertion 3) :

\section{Theorem :}

- Let $P$ be an arbitrary finite direct product of groups of order 2 and dihedral 2-groups. Then the Dade group of any factor group of $P$ is torsion free.

- Let $n$ be a positive integer. For any integer $m \geq 4$, let $P=S D_{2^{m}}$ be a semidihedral group of order $2^{m}$, and let $P^{* n}$ denote the central product of $n$-copies of $P$. Then the torsion part of the Dade group of $P^{* n}$ is isomorphic to $(\mathbb{Z} / 2 \mathbb{Z})^{2^{(n-1)(m-3)}}$. 
This also yields similar results on groups of units of Burnside rings of these groups (Remark 5.39), or on representations of central products of $p$-groups, as in Examples 5.34 and 5.35:

Example : Let $p$ be a prime, let $X$ be an extraspecial p-group, and let $Q$ be a non-trivial p-group. Let $K$ be a field of characteristic 0 . Then $Q$ has the same number (possibly 0) of isomorphism classes of faithful irreducible representations over $K$ as any central product $X * Q$.

Another possibly interesting phenomenon is that some non-isomorphic $p$-groups may become isomorphic in the category $\mathcal{R}_{p}$. This means that some non-isomorphic $p$-groups cannot be distinguished using only rational $p$-biset functors. When $p=2$, there are even examples where this occurs for groups of different orders (Example 5.16). When $p>2$, saying that the $p$-groups $P$ and $Q$ are isomorphic in $\mathcal{R}_{p}$ is equivalent to saying that the group algebras $\mathbb{Q} P$ and $\mathbb{Q} Q$ have isomorphic centers (Proposition 5.17).

The category $\mathcal{R}_{p}$ is built as follows : consider first the category $\mathcal{R}_{p}^{\sharp}$, which is the quotient category of the biset category of finite $p$-groups (in which objects are finite $p$-groups and morphisms are virtual bisets) obtained by killing a specific element $\delta$ in the Burnside group of the Sylow $p$-subgroup of $P G L\left(3, \mathbb{F}_{p}\right)$. Then take idempotent completion, and additive completion of the resulting category.

In particular, this construction relies on bisets, and related functors. Consequently, the paper is organized as follows : Section 2 is a (not so) quick summary of the background on biset functors, Roquette groups, genetic bases of $p$-groups, and rational $p$-biset functors. The category $\mathcal{R}_{p}$ is introduced in Section 3, and in Section 4, its tensor structure is described. Finally Section 5 gives some examples and applications.

Acknowledgments : Even though the idea of considering the category $\mathcal{R}_{p}$ was implicit in [7], the present paper wouldn't exist without the illuminating conversations I had with Paul Balmer in november 2010, during which I gradually understood I was a kind of Monsieur Jourdain of tensor categories... I wish to thank him for this revelation.

\section{Rational $p$-biset functors}

2.1. Biset functors. The biset category $\mathcal{C}$ of finite groups is defined as follows :

- The objects of $\mathcal{C}$ are the finite groups. 
- Let $G$ and $H$ be finite groups. Then

$$
\operatorname{Hom}_{\mathcal{C}}(G, H)=B(H, G),
$$

where $B(H, G)$ denotes the Grothendieck group of the category of finite $(H, G)$-bisets, i.e. the Burnside group of the group $H \times G^{o p}$.

- Let $G, H$, and $K$ be finite groups. The composition of morphisms

$$
B(K, H) \times B(H, G) \rightarrow B(K, G)
$$

in the category $\mathcal{C}$ is the linear extension of the product induced by the product of bisets $(V, U) \mapsto V \times_{H} U$, where $V$ is a $(K, H)$-biset, and $U$ is an $(H, G)$-biset.

- The identity morphism of the finite group $G$ is the image in $B(G, G)$ of the set $G$, endowed with its $(G, G)$-biset structure given by left and right multiplication.

2.2. Definition : $A$ biset functor is an additive functor from $\mathcal{C}$ to the category of abelian groups. A morphism of biset functors is a natural transformation of functors.

Morphisms of biset functors can be composed, and the resulting category of biset functors is denoted by $\mathcal{F}$. It is an abelian category.

\subsection{Examples :}

1. The correspondence $B$ sending a finite group $G$ to its Burnside group $B(G)$ is a biset functor, called the Burnside functor : indeed $B(G)=$ $\operatorname{Hom}_{\mathcal{C}}(\mathbf{1}, G)$, so $B$ is in fact the Yoneda functor $\operatorname{Hom}_{\mathcal{C}}(\mathbf{1},-)$.

2. The formalism of bisets gives a single framework for the usual operations of induction, restriction, inflation, deflation, and transport by isomorphism via the following correspondences :

- If $H$ is a subgroup of $G$, then let $\operatorname{Ind}_{H}^{G} \in B(G, H)$ denote the set $G$, with left action of $G$ and right action of $H$ by multiplication.

- If $H$ is a subgroup of $G$, then let $\operatorname{Res}_{H}^{G} \in B(H, G)$ denote the set $G$, with left action of $H$ and right action of $G$ by multiplication.

- If $N \unlhd G$, and $H=G / N$, then let $\operatorname{Inf}_{H}^{G} \in B(G, H)$ denote the set $H$, with left action of $G$ by projection and multiplication, and right action of $H$ by multiplication.

- If $N \unlhd G$, and $H=G / N$, then let $\operatorname{Def}_{H}^{G} \in B(H, G)$ denote the set $H$, with left action of $H$ by multiplication, and right action of $G$ by projection and multiplication. 
- If $\varphi: G \rightarrow H$ is a group isomorphism, then let $\operatorname{Iso}_{G}^{H}=\operatorname{Iso}_{G}^{H}(\varphi) \in$ $B(H, G)$ denote the set $H$, with left action of $H$ by multiplication, and right action of $G$ by taking image by $\varphi$, and then multiplying in $H$.

- When $H$ is a subgroup of $G$, let $\operatorname{Defres}_{N_{G}(H) / H}^{G} \in B\left(N_{G}(H) / H, G\right)$ denote the set $H \backslash G$, viewed as a $\left(N_{G}(H) / H, G\right)$-biset. It is equal to the composition $\operatorname{Def}_{N_{G}(H) / H}^{G} \circ \operatorname{Res}_{N_{G}(H)}^{G}$.

- When $H$ is a subgroup of $G$, let $\operatorname{Indinf}_{N_{G}(H) / H}^{G} \in B\left(G, N_{G}(H) / H\right)$ denote the set $G / H$, viewed as a $\left(G, N_{G}(H) / H\right)$-biset. It is equal to the composition $\operatorname{Ind}_{N_{G}(H)}^{G} \circ \operatorname{Inf}_{N_{G}(H) / H}^{N_{G}(H)}$.

2.4. $p$-biset functors. From now on, the symbol $p$ will denote a prime number.

\subsection{Definition and Notation :}

- The biset category $\mathcal{C}_{p}$ of finite p-groups is the full subcategory of $\mathcal{C}$ consisting of finite p-groups.

- A p-biset functor is an additive functor from $\mathcal{C}_{p}$ to the category of abelian groups. A morphism of p-biset functors is a natural transformation of functors.

- $p$-biset functors form an abelian category $\mathcal{F}_{p}$.

\subsection{Roquette $p$-groups.}

2.7. Definition : A finite group $G$ is call a Roquette group if it has normal rank 1, i.e. if all the normal abelian subgroups of $G$ are cyclic.

The Roquette $p$-groups have been first classified by... Roquette ([14], see also [12]) : these are the cyclic groups, if $p>2$. The Roquette 2-groups are the cyclic groups, the generalized quaternion groups, the dihedral and semidihedral groups of order at least 16 .

More generally, the $p$-hyperelementary Roquette groups have been classified by Hambleton, Taylor and Williams (Theorem 3.A.6 of [13]).

The following schematic diagram represents the lattice of subgroups of the dihedral group $D_{16}$, the quaternion group $Q_{16}$, and the semi-dihedral group $S D_{16}$ (an horizontal dotted link between two vertices means that the 
corresponding subgroups are conjugate) :
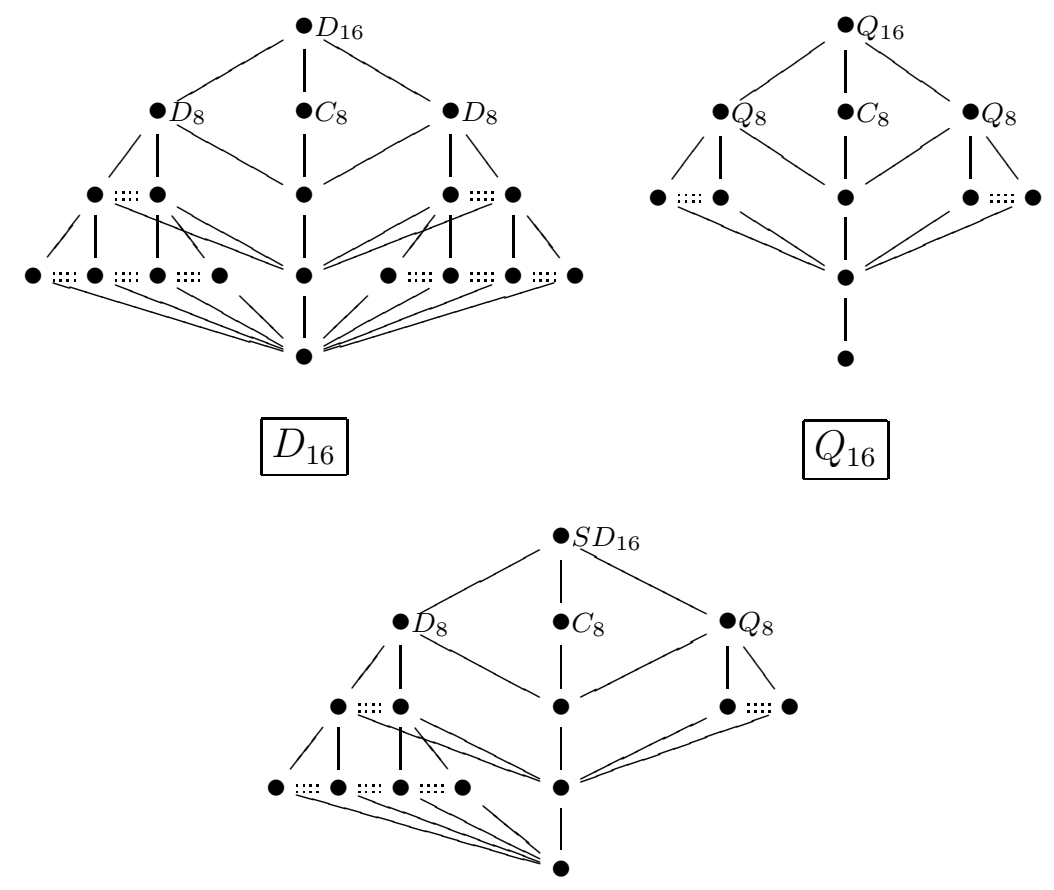

$Q_{16}$

$$
S D_{16}
$$

These diagrams give a good idea of the general case.

2.8. Definition : Let $G$ be a finite group, of exponent e. An axis of $G$ is a cyclic subgroup of order e in $G$. An axial subgroup of $G$ is a subgroup of an axis of $G$.

With these definitions, let us recall without proof the following properties of Roquette $p$-groups :

2.9. Lemma : Let $P$ be a non-trivial Roquette p-group, of exponent $e_{P}$.

1. The center of $P$ is cyclic, hence $P$ admits a unique central subgroup $Z_{P}$ of order $p$.

2. There exists a non-trivial subgroup $Q$ of $P$ such that $Q \cap Z(P)=\mathbf{1}$ if and only if $p=2$, and $P$ is dihedral or semidihedral. In this case moreover $|Q|=2$, and $N_{P}(Q)=Q Z_{P}$.

3. If $P$ is not cyclic, then $p=2$ and $e_{P}=|P| / 2$.

4. There is a unique axis in $P$, except in the case $P \cong Q_{8}$, where there are three of them. Any axis of $P$ is normal in $P$. 
5. If $R$ is a non-trivial axial subgroup of $P$, then $R \geq Z_{P}$, and $R \unlhd P$. If moreover $|R| \geq p^{2}$, then $C_{P}(R)$ is the only axis of $P$ containing $R$.

Let us also recall the following :

2.10. Lemma : Let $P$ be a finite Roquette -group. Then there is a unique simple faithful $\mathbb{Q} P$-module $\Phi_{P}$, up to isomorphism.

Proof : See [8] Proposition 9.3.5.

2.11. Example : Let $P$ be a cyclic group of order $p^{m}$, and suppose first that $m>1$. The algebra $\mathbb{Q} P$ is isomorphic to the algebra $A=\mathbb{Q}[X] /\left(X^{p^{m}}-1\right)$. As

$$
X^{p^{m}}-1=\left(X^{p^{m-1}}-1\right) \Psi_{p^{m}}(X),
$$

where $\Psi_{p^{m}}$ denotes the $p^{m}$-th cyclotomic polynomial, it follows that there is a split exact sequence of $A$-modules

$$
0 \mapsto \mathbb{Q}[X] /\left(\Psi_{p^{m}}\right) \rightarrow A \rightarrow \mathbb{Q}[X] /\left(X^{p^{m-1}}-1\right) \rightarrow 0
$$

which can be viewed as the following sequence of $\mathbb{Q} P$-modules

$$
0 \rightarrow \Phi_{P} \rightarrow \mathbb{Q} P \rightarrow \mathbb{Q}(P / Z) \rightarrow 0
$$

where $Z$ is the unique subgroup of order $p$ of $P$. It follows that there is an isomorphism of $\mathbb{Q}$-algebras

$$
\operatorname{End}_{\mathbb{Q} P} \Phi_{P} \cong \mathbb{Q}[X] /\left(\Psi_{p^{m}}\right) \cong \mathbb{Q}\left(\zeta_{p^{m}}\right)
$$

where $\zeta_{p^{m}}$ is a primitive $p^{m}$-th root of unity in $\mathbb{C}$.

Now if $m=0$, then $P=\mathbf{1}, \Phi_{P}=\mathbb{Q}$, and $\operatorname{End}_{\mathbb{Q} P} \Phi_{P} \cong \mathbb{Q}$, also.

\subsection{Expansive and genetic subgroups.}

2.13. Definition : A subgroup $H$ of a group $G$ is called expansive if for any $g \in G$ such that $H^{g} \neq H$, the group $\left(H^{g} \cap N_{G}(H)\right) H / H$ contains a non trivial normal subgroup of $N_{G}(H) / H$, i.e. if

$$
g \in G-N_{G}(H) \Longrightarrow \bigcap_{n \in N_{G}(H)}\left(H^{g n} \cap N_{G}(H)\right) H>H .
$$


2.14. Example : If $H \unlhd G$, then $H$ is expansive in $G$. More generally, if $N_{G}(H) \unlhd G$, then $H$ is expansive in $G$ : indeed $N_{G}\left(H^{g}\right)=N_{G}(H)$, for any $g \in G$. Hence for $g \in G-N_{G}(H)$

$$
\bigcap_{n \in N_{G}(H)}\left(H^{g n} \cap N_{G}(H)\right) H=\left(H^{g} \cap N_{G}(H)\right) H=H^{g} \cdot H>H .
$$

2.15. Notation : When $H$ is a subgroup of the group $G$, denote by $Z_{G}(H)$ the subgroup of $N_{G}(H)$, containing $H$, defined by

$$
Z_{G}(H) / H=Z\left(N_{G}(H) / H\right)
$$

The following is an easy consequence of well known properties of $p$ groups :

2.16. Lemma : [8] Lemma 9.5.2] Let $Q$ be a subgroup of a finite p-group $P$. Then $Q$ is expansive in $P$ if and only if

$$
\forall g \in P, \quad Q^{g} \cap Z_{P}(Q) \leq Q \Longrightarrow Q^{g}=Q
$$

2.17. Example : Let $P$ be a p-group, and let

$$
\Delta(P)=\{(x, x) \in(P \times P) \mid x \in P\}
$$

denote the diagonal subgroup of $(P \times P)$. Then $N_{P \times P}(\Delta(P)) / \Delta(P) \cong Z(P)$, and $\Delta(P)$ is expansive in $(P \times P)$ if and only if

$$
\forall x \in P, \quad[P, x] \cap Z(P)=\{1\} \Longrightarrow x \in Z(P)
$$

where $[P, x]$ is the set of commutators $[y, x]=y^{-1} x^{-1} y x=y^{-1} y^{x}$, for $y \in P$. In particular, if $[P, P] \leq Z(P)$, then $\Delta(P)$ is expansive in $(P \times P)$.

Proof : Indeed $N_{P \times P}(\Delta(P))$ consists of pairs $(a, b) \in(P \times P)$ such that $a b^{-1} \in Z(P)$. This shows that $N_{P \times P}(\Delta(P)) / \Delta(P) \cong Z(P)$, and that

$$
Z_{P \times P}(\Delta(P))=N_{P \times P}(\Delta(P))=(\mathbf{1} \times Z(P)) \Delta(P) .
$$

Now $\Delta(P)^{(u, v)}=\Delta(P)^{(1, x)}$, for any $(u, v) \in(P \times P)$, where $x=u^{-1} v$, and

$$
\Delta(P)^{(1, x)} \cap Z_{P \times P}(\Delta(P))=\left\{\left(t, t^{x}\right) \mid t \in P, t^{-1} t^{x} \in Z(P)\right\} .
$$


Hence $\Delta(P)^{(1, x)} \cap Z_{P \times P}(\Delta(P)) \leq \Delta(P)$ if and only if for any $t \in P$, the assumption $t^{-1} t^{x} \in Z(P)$ implies $t=t^{x}$, i.e. $[t, x]=1$, in other words if $[P, x] \cap Z(P)=\{1\}$. Hence $\Delta(P)$ is expansive in $(P \times P)$ if and only if for any $x \in P$, the assumption $[P, x] \cap Z(P)=\{1\}$ implies $(1, x) \in N_{P \times P}(\Delta(P))$, i.e. $x \in Z(P)$, as claimed. The last assertion follows trivially.

2.18. Definition : Let $Q$ be a subgroup of the finite $p$-group $P$. Then $Q$ is called a genetic subgroup of $P$ if $Q$ is expansive in $P$ and if the group $N_{P}(Q) / Q$ is a Roquette group.

2.19. Definition : Define a relation $\widehat{-}_{P}$ on the set of subgroups of the finite p-group $P$ by

$$
Q \widehat{=}_{P} R \Leftrightarrow \exists g \in P, \quad Q^{g} \cap Z_{P}(R) \leq R \text { and }{ }^{g} R \cap Z_{P}(Q) \leq Q .
$$

2.20. Lemma : Let $P$ be a finite p-group. If $Q$ and $R$ are subgroups of $P$ such that $N_{P}(Q)=N_{P}(R) \unlhd P$, then

$$
Q \widehat{=}_{P} R \Leftrightarrow Q={ }_{P} R
$$

Proof : Indeed, since $P$ is a $p$-group, saying that $Q^{g} \cap Z_{P}(R) \leq R$ is equivalent to saying that the subgroup $\left(Q^{g} \cap N_{P}(R)\right) R / R$ of $N_{P}(R) / R$ contains no non-trivial normal subgroup of $N_{P}(R) / R$, i.e. that

$$
\bigcap_{n \in N_{P}(R)}\left(Q^{g n} \cap N_{P}(R)\right) R=R .
$$

But if $N_{P}(Q) \unlhd P$, then $N_{P}(Q)=N_{P}\left(Q^{g}\right)=N_{P}(R)$, for any $g \in G$. Hence

$$
\bigcap_{n \in N_{G}(R)}\left(Q^{g n} \cap N_{P}(R)\right) R=Q^{g} \cdot R .
$$

This is equal to $R$ if and only if $Q^{g} \leq R$. Similarly ${ }^{g} R \cap Z_{P}(Q) \leq Q$ if and only if ${ }^{g} R \leq Q$. Hence $Q^{g}=R$.

2.21. Definition : Let $G$ be a group.

1. A section of $G$ is a pair $(T, S)$ of subgroups of $G$ such that $S \unlhd T$. The 
quotient $T / S$ is called the corresponding subquotient of $G$.

2. Two sections $(T, S)$ and $(Y, X)$ of $G$ are said to be linked (notation $(T, S)-(Y, X))$ if

$$
S(T \cap Y)=T, \quad X(T \cap Y)=Y, \quad T \cap X=S \cap Y .
$$

They are said to be linked modulo $G$ (notation $(T, S)-_{G}(Y, X)$ ) if there exists $g \in G$ such that $(T, S)-\left({ }^{g} Y,{ }^{g} X\right)$.

Observe in particular that if $(T, S)-_{G}(Y, X)$, then the corresponding subquotients $T / S$ and $Y / X$ are isomorphic.

2.22. Theorem : Let $P$ be a finite p-group.

1. If $S$ is a genetic subgroup of $P$, then the module

$$
V(S)=\operatorname{Ind}_{N_{P}(S)}^{P} \operatorname{Inf}_{N_{P}(S) / S}^{N_{P}(S)} \Phi_{N_{P}(S) / S}
$$

is a simple $\mathbb{Q} P$-module. Moreover, the functor $\operatorname{Ind}_{N_{P}(S)}^{P} \operatorname{Inf}_{N_{P}(S) / S}^{N_{P}(S)}$ induces an isomorphism of $\mathbb{Q}$-algebras

$$
\operatorname{End}_{Q P} V(S) \cong \operatorname{End}_{\mathbb{Q} N_{P}(S) / S} \Phi_{N_{P}(S) / S}
$$

2. If $V$ is a simple $\mathbb{Q} P$-module, then there exists a genetic subgroup $S$ of $P$ such that $V \cong V(S)$.

3. If $S$ and $T$ are genetic subgroups of $P$, then

$$
V(S) \cong V(T) \Leftrightarrow S \widehat{=}_{P} T \Leftrightarrow\left(N_{P}(S), S\right)-_{P}\left(N_{P}(T), T\right) .
$$

In particular, if $S \widehat{=}_{P} T$, then $N_{P}(S) / S \cong N_{P}(T) / T$. Moreover, the relation $\widehat{C}_{P}$ is an equivalence relation on the set of genetic subgroups of $P$, and the corresponding set of equivalence classes is in one to one correspondence with the set of isomorphism classes of simple $\mathbb{Q} P$ modules.

Proof : See [8], Theorem 9.6.1.

2.23. Definition : Let $P$ be a finite p-group. A genetic basis of $P$ is a set of representatives of equivalence classes of genetic subgroups of $P$ for the relation $\widehat{ }_{P}$. 
2.24. Faithful elements. ([8] Sections 6.2 and 6.3) Let $G$ be a finite group. If $N$ is a normal subgroup of $G$, recall from Examples 2.3 that $\operatorname{Inf}_{G / N}^{G}$ denotes the set $G / N$, viewed as a $(G, G / N)$-biset for the actions given by (projection to the factor group and) multiplication in $G / N$. Similarly $\operatorname{Def}_{G / N}^{G}$ denotes the same set $G / N$, considered as a $(G / N, G)$-biset.

There is an isomorphism of $(G / N, G / N)$-bisets

$$
\operatorname{Id}_{G / N} \cong \operatorname{Def}_{G / N}^{G} \circ \operatorname{Inf}_{G / N}^{G}
$$

More generally, if $M$ and $N$ are normal subgroups of $G$, there is a isomorphism of $(G / M, G / N)$-bisets

$$
\operatorname{Def}_{G / M}^{G} \circ \operatorname{Inf}_{G / N}^{G}=\operatorname{Inf}_{G / M N}^{G / M} \circ \operatorname{Def}_{G / M N}^{G / N} .
$$

It follows that if $j_{N}^{G}$ is defined by

$$
j_{N}^{G}=\operatorname{Inf}_{G / N}^{G} \circ \operatorname{Def}_{G / N}^{G},
$$

then $j_{M}^{G} \circ j_{N}^{G}=j_{M N}^{G}$. In particular $j_{N}^{G}$ is an idempotent of $B(G, G)$. Moreover, by a standard orthogonalization procedure, the elements $f_{N}^{G}$ defined for $N \unlhd G$ by

$$
f_{N}^{G}=\sum_{N \leq M \unlhd G} \mu_{\unlhd G}(N, M) j_{M}^{G}
$$

where $\mu_{\unlhd G}(N, M)$ is the Möbius function of the poset of normal subgroups of $G$, are orthogonal idempotents of $B(G, G)$, and their sum is equal to $\operatorname{Id}_{G / N}$. The idempotent $f_{1}^{G}$ is of special importance :

2.27. Lemma : Let $G$ be a finite group, and $N$ be a normal subgroup of $G$.

1. $f_{N}^{G}=\operatorname{Inf}_{G / N}^{G} \circ f_{\mathbf{1}}^{G / N} \circ \operatorname{Def}_{G / N}^{G}$ in $B(G, G)$.

2. If $N \neq 1$, then $\operatorname{Def}_{G / N}^{G} \circ f_{\mathbf{1}}^{G}=0$ in $B(G / N, G)$, and $f_{\mathbf{1}}^{G} \circ \operatorname{Inf}_{G / N}^{G}=0$ in $B(G, G / N)$.

Proof : Assertion 1 is Remark 6.2.9 of [8], and Assertion 2 is a special case of Proposition 6.2.6.

If $F$ is a biset functor, the set $\partial F(G)$ of faithful elements of $F(G)$ is defined by

$$
\partial F(G)=F\left(f_{1}^{G}\right) F(G) .
$$

It can be shown ([8] Lemma 6.3.2) that

$$
\partial F(G)=\bigcap_{\mathbf{1}<N \unlhd G} \operatorname{Ker} F\left(\operatorname{Def}_{G / N}^{G}\right)
$$


2.28. Example : Let $F=R_{K}$ be the representation functor over a field $K$ of characteristic 0 . Then for a finite group $G$, the group $\partial R_{K}(G)$ is the direct summand of $R_{K}(G)$ with basis the set of (isomorphism classes of) faithful irreducible $K G$-modules.

2.29. Lemma : Let $G$ be a group, and $S$ be a subgroup of $G$, such that $S \cap Z(G) \neq 1$. Then $\operatorname{Defres}_{N_{G}(S) / S}^{G} f_{1}^{G}=0$ in $B\left(N_{G}(S) / S, G\right)$.

Proof : Set $N=S \cap Z(G), \bar{G}=G / N$, and $\bar{S}=S / N$. Then

$$
\operatorname{Defres}_{N_{G}(S) / S}^{G}=\operatorname{Defres}_{\left.N_{\bar{G}} \bar{G}\right) / \bar{S}}^{\overline{D^{\prime}}} \operatorname{Def}_{G / N}^{G}
$$

Now $\operatorname{Def}_{G / N}^{G} f_{1}^{G}=0$ if $N \neq 1$, by Lemma 2.27 .

2.30. Theorem : [8] Theorem 10.1.1] Let $P$ be a finite p-group, and $\mathcal{B}$ be a genetic basis of $P$. Then, for any $p$-biset functor $F$, the map

$$
\mathcal{I}_{\mathcal{B}}=\underset{S \in \mathcal{B}}{\oplus} \operatorname{Indinf}_{N_{P}(S) / S}^{P}: \underset{S \in \mathcal{B}}{\oplus} \partial F\left(N_{P}(S) / S\right) \rightarrow F(P)
$$

is split injective. A left inverse is the map

$$
\mathcal{D}_{\mathcal{B}}=\underset{S \in \mathcal{B}}{\oplus} f_{\mathbf{1}}^{N_{P}(S) / S} \circ \operatorname{Defres}_{N_{P}(S) / S}^{P}: F(P) \rightarrow \underset{S \in \mathcal{B}}{\bigoplus} \partial F\left(N_{P}(S) / S\right)
$$

One can show ([8] Lemma 10.1.2) that if $\mathcal{B}$ and $\mathcal{B}^{\prime}$ are genetic bases of $P$, the map $\mathcal{I}_{\mathcal{B}}$ is an isomorphism if and only if the map $\mathcal{I}_{\mathcal{B}^{\prime}}$ is an isomorphism. This motivates the following definition :

2.31. Definition : A p-biset functor $F$ is called rational if for any finite p-group $P$, there exists a genetic basis $\mathcal{B}$ of $P$ such that the map $\mathcal{I}_{\mathcal{B}}$ is an isomorphism.

So $F$ is rational if and only if for any finite $p$-group $P$ and any genetic basis $\mathcal{B}$ of $P$, the map $\mathcal{I}_{\mathcal{B}}$ is an isomorphism.

\subsection{Examples :}

- The functor $R_{\mathbb{Q}}$ of rational representations, which sends the finite $p$-group $P$ to the group $R_{\mathbb{Q}}(P)$, is a rational $p$-biset functor. This example is of course the reason for calling rational the $p$-biset functors of Definition 2.31. This 
choice has proved rather unfortunate, since the $p$-biset functor $R_{\mathbb{C}}$ of complex representations is also a rational functor... More generally, if $K$ is a field of characteristic 0 , then the functor $R_{K}$ is a rational $p$-biset functor.

- The functor of units of the Burnside ring, sending a $p$-group $P$ to the group of units $B^{\times}(P)$ of its Burnside ring, is a rational $p$-biset functor (see [6]).

- Let $k$ be a field of characteristic $p$. The correspondence sending a finite $p$-group $P$ to the torsion part $D_{k}^{t}(P)$ of the Dade group of $P$ over $k$ is not a biset functor in general, because of phenomenons of Galois twists, but still the maps $\mathcal{I}_{\mathcal{B}}$ and $\mathcal{D}_{\mathcal{B}}$ can be defined for $D_{k}^{t}$, and Theorem 2.30 holds (see [5]).

\section{The Roquette category}

3.1. Notation : Let $\pi$ be a projective plane over $\mathbb{F}_{p}$, and let $X$ denote a Sylow p-subgroup of $\operatorname{Aut}(\pi) \cong \operatorname{PGL}\left(3, \mathbb{F}_{p}\right)$. Let $\mathbb{L}$ be the set of lines of $\pi$, and let $\mathbb{P}$ be the set of points of $\pi$, both viewed as elements of $B(X)$. Let $\delta=\mathbb{L}-\mathbb{P} \in B(X)$. Equivalently

$$
\delta=(X / I-X / I Z)-(X / J-X / J Z)
$$

where $I$ and $J$ are non-conjugate non-central subgroups of order $p$ of $X$, and $Z$ is the center of $X$.

Let $B_{\delta}$ denote the p-biset subfunctor of $B$ generated by $\delta$.

3.2. Remark : When $p=2$, the group $X$ is dihedral of order 8 , and $\delta$ is well-defined up to a sign. When $p>2$, the group $X$ is an extraspecial $p$-group of order $p^{3}$ and exponent $p$, and there are several possible choices for the element $\delta$. However, in any case, the functor $B_{\delta}$ does not depend on the choice of $\delta$.

3.3. Definition : The Roquette category $\mathcal{R}_{p}$ of finite $p$-groups is defined as the idempotent additive completion of the category $\mathcal{R}_{p}^{\sharp}$, quotient of the biset category $\mathcal{C}_{p}$, defined as follows :

1. the objects of $\mathcal{R}_{p}^{\sharp}$ are the finite p-groups.

2. if $P$ and $Q$ are finite $p$-groups, then

$$
\operatorname{Hom}_{\mathcal{R}_{p}^{\sharp}}(P, Q)=\left(B / B_{\delta}\right)(Q, P)
$$


is the quotient of $B\left(Q \times P^{o p}\right)$ by $B_{\delta}\left(Q \times P^{o p}\right)$.

3. the composition in $\mathcal{R}_{p}^{\sharp}$ is induced by the composition of bisets.

4. the identity morphism of the finite $p$-group $P$ in $\mathcal{R}_{p}^{\sharp}$ is the image of $\operatorname{Id}_{P}$ in $\left(B / B_{\delta}\right)(P, P)$.

3.4. Remark : It was shown in [7] that $\mathcal{R}_{p}^{\sharp}$ is indeed a category. It was also shown there that if $p>2$, the functor $B_{\delta}$ is equal to the kernel $K$ of the linearization morphism $B \rightarrow R_{\mathbb{Q}}$. It follows that in this case, for any two finite $p$-groups $P$ and $Q$

$$
\operatorname{Hom}_{\mathcal{R}_{p}}(P, Q) \cong R_{\mathbb{Q}}\left(Q \times P^{o p}\right)
$$

is isomorphic to the Grothendieck group of $(\mathbb{Q} Q, \mathbb{Q} P)$-bimodules, or, equivalently by the Ritter-Segal theorem, to the Grothendieck group of the subcategory of $(\mathbb{Q} Q, \mathbb{Q} P)$-permutation bimodules. In other words in this case, the category $\mathcal{R}_{p}$ is the full subcategory category of the category considered by Barker in [1], consisting of finite $p$-groups. The construction of the category $\mathcal{R}_{p}$ is also very similar to the construction of the category $\mathbb{Q} G$-Morita by Hambleton, Taylor, and Williams in [13] (Definition 1.A.4).

In the case $p=2$, the situation is more complicated : the functor $B_{\delta}$ is a proper subfunctor of the kernel $K$, and there is a short exact sequence

$$
0 \rightarrow K / B_{\delta} \rightarrow B / B_{\delta} \rightarrow R_{\mathbb{Q}} \rightarrow 0
$$

of $p$-biset functors. Moreover, for each $p$-group $P$, the group $\left(K / B_{\delta}\right)(P)$ is a finite elementary abelian 2-group of rank equal to the number of groups $S$ in a genetic basis of $P$ for which $N_{P}(S) / S$ is dihedral.

3.5. Lemma : The direct product $(P, Q) \mapsto P \times Q$ of p-groups induces a well defined symmetric monoidal structure on $\mathcal{R}_{p}^{\sharp}$.

Proof : Let $P, P^{\prime}, Q$ and $Q^{\prime}$ be finite $p$-groups. If $U$ is a finite $\left(P^{\prime}, P\right)$-biset and $V$ is a finite $\left(Q^{\prime}, Q\right)$-biset, then $U \times V$ is a $\left(P^{\prime} \times Q^{\prime}, P \times Q\right)$-biset. This induces a bilinear map

$$
\pi: B\left(P^{\prime}, P\right) \times B\left(Q^{\prime}, Q\right) \rightarrow B\left(P^{\prime} \times Q^{\prime}, P \times Q\right),
$$

and this clearly induces a symmetric monoidal structure on the biset category $\mathcal{C}_{p}$. This induces a monoidal structure on the quotient category if

$$
\pi\left(B_{\delta}\left(P^{\prime}, P\right), B\left(Q^{\prime}, Q\right)\right) \subseteq B_{\delta}\left(P^{\prime} \times Q^{\prime}, P \times Q\right)
$$


But this is a consequence of the following : let $X$ be as defined in Notation 3.1, let $U$ be a finite $\left(P^{\prime}, P \times X\right)$-set, let $D$ be an $X$-set, and $V$ be a finite $\left(Q^{\prime}, Q\right)$ biset. Clearly, there is an isomorphism of $\left(P^{\prime} \times Q^{\prime}, P \times Q\right)$-sets

$$
\left(U \times_{X} D\right) \times V \cong(U \times V) \times_{X} D
$$

where the right action of $X$ on $U \times V$ is defined in the obvious way

$$
\forall(u, v) \in U \times V, \forall x \in X, \quad(u, v) x=(u x, v) .
$$

The lemma follows.

3.6. Recall that the objects of the idempotent additive completion $\mathcal{R}_{p}$ are by definition formal finite sums $\underset{(P, e) \in \mathcal{P}}{\oplus}(P, e)$ of pairs of the form $(P, e)$, where $P$ is a finite $p$-group, and $e$ is an idempotent in $\operatorname{Hom}_{\mathcal{R}_{p}^{\sharp}}(P, P)=\left(B / B_{\delta}\right)(P, P)$. A morphism $\varphi: \underset{(P, e) \in \mathcal{P}}{\oplus}(P, e) \rightarrow \underset{(Q, f) \in \mathcal{Q}}{\oplus}(Q, f)$ in $\mathcal{R}_{p}$ is a matrix indexed by $\mathcal{P} \times \mathcal{Q}$, where the coefficient $\varphi_{(P, e),(Q, f)}$ belongs to $f \operatorname{Hom}_{\mathcal{R}_{p}^{\sharp}}(P, Q) e$. The composition of morphisms is given by matrix multiplication. In particular :

\subsection{Definition and Notation : Let $P$ be a finite $p$-group.}

1. The object $\left(P, \operatorname{Id}_{P}\right)$ of $\mathcal{R}_{p}$ is denoted by $P$. Similarly, when $Q$ is a finite p-group, and $f \in B(Q, P)$, the corresponding morphism from $\left(P, \operatorname{Id}_{P}\right)$ to $\left(Q, \operatorname{Id}_{P}\right)$ in the category $\mathcal{R}_{p}$ is simply denoted by $f$.

2. The edge $\partial P$ of $P$ is the pair $\left(P, f_{1}^{P}\right)$ of $\mathcal{R}_{p}$.

The category of additive functors from $\mathcal{R}_{p}$ to abelian groups is equivalent to the category of additive functors from $\mathcal{R}_{p}^{\sharp}$ to abelian groups. It was shown in [7] that the latter is exactly the category of rational $p$-biset functors. If $F^{\sharp}$ is such a functor, then $F^{\sharp}$ extends to a functor $F$ on $\mathcal{R}_{p}$ defined as follows :

$$
F(\underset{(P, e) \in \mathcal{P}}{\oplus}(P, e))=\underset{(P, e) \in \mathcal{P}}{\bigoplus} F^{\sharp}(e)\left(F^{\sharp}(P)\right),
$$

with the obvious definition of $F(\varphi)$ for a morphism $\varphi$ in the category $\mathcal{R}_{p}$. In particular, with the above notation

$$
F(\partial P)=\partial F^{\sharp}(P)
$$

In the sequel, we will use the same symbol for $F$ and $F^{\sharp}$, writing in particular $F(\partial P)=\partial F(P)$. 
3.8. Proposition : Let $P$ be a finite p-group. Then

$$
P \cong \underset{N \unlhd P}{\oplus} \partial(P / N)
$$

in the category $\mathcal{R}_{p}$.

Proof : Let

$$
a: P \rightarrow \underset{N \unlhd P}{\oplus} \partial(P / N)
$$

be the direct sum of the morphisms induced by the elements $f_{\mathbf{1}}^{P / N} \operatorname{Def}_{P / N}^{P}$ of $B(P / N, P)$, and let

$$
b: \underset{N \unlhd P}{\oplus} \partial(P / N) \rightarrow P
$$

be defined similarly from the elements $\operatorname{Inf}_{P / N}^{P} f_{\mathbf{1}}^{P / N}$ of $B(P, P / N)$.

By Lemma 2.27

$$
\sum_{N \unlhd P} \operatorname{Inf}_{P / N}^{P} f_{\mathbf{1}}^{P / N} \operatorname{Def}_{P / N}^{P}=\sum_{N \unlhd P} f_{N}^{P}=\operatorname{Id}_{P}
$$

in $B(P, P)$, thus $a \circ b$ is equal to the identity morphism of $P$ in $\mathcal{R}_{P}$. Conversely, for normal subgroups $N$ and $M$ of $P$

$$
f_{\mathbf{1}}^{P / N} \operatorname{Def}_{P / N}^{P} \operatorname{Inf}_{P / M}^{P} f_{\mathbf{1}}^{P / M}=f_{\mathbf{1}}^{P / N} \operatorname{Inf}_{P / N M}^{P / N} \operatorname{Def}_{P / N M}^{P / M} f_{\mathbf{1}}^{P / M},
$$

by Equation 2.25. This is equal to 0 if $N \neq M$, by Lemma 2.27. And if $N=M$, this is equal to $f_{\mathbf{1}}^{P / N}$. It follows that $b \circ a$ is equal to the identity morphism of $\underset{N \unlhd P}{\oplus} \partial(P / N)$, and this completes the proof.

3.9. Corollary : If $P$ is non-trivial, with cyclic center, then

$$
P \cong \partial P \oplus(P / Z)
$$

in $\mathcal{R}_{p}$, where $Z$ is the unique central subgroup of order $p$ in $P$.

Proof : Indeed, if $N$ is a non-trivial normal subgroup of $P$, then $N \geq Z$. Thus

$$
P \cong \partial P \oplus \underset{N \geq Z}{\oplus} \partial(P / N) \cong \partial P \oplus(P / Z)
$$

in the category $\mathcal{R}_{p}$. 
3.10. Remark : More generally, let $P$ be a finite $p$-group, and let $N$ be a normal subgroup of $P$. Since in $\mathcal{R}_{p}$

$$
P / N \cong \underset{N \leq M \unlhd P}{\oplus} \partial((P / N) /(M / N)) \cong \underset{N \leq M \unlhd P}{\oplus} \partial(P / M)
$$

it follows that $P / N$ is isomorphic to a direct summand of $P$ in the category $\mathcal{R}_{p}$.

\subsection{Theorem :}

1. The Roquette category $\mathcal{R}_{p}$ is an additive tensor category.

2. Let $P$ be a finite p-group, and $\mathcal{B}$ be a genetic basis of $P$. Then, in the category $\mathcal{R}_{p}$,

$$
P \cong \underset{S \in \mathcal{B}}{\oplus} \partial \bar{N}_{P}(S)
$$

where $\bar{N}_{P}(S)=N_{P}(S) / S$.

3. Let $P$ be a finite p-group, and $\mathcal{B}$ be a genetic basis of $P$. Then, in the category $\mathcal{R}_{p}$,

$$
\partial P \cong \underset{\substack{S \in \mathcal{B} \\ S \cap Z(P)=\mathbf{1}}}{\bigoplus} \partial \bar{N}_{P}(S)
$$

Proof : Assertion 1 results from standard results : in particular, the tensor product of $\underset{(P, e) \in \mathcal{P}}{\oplus}(P, e)$ and $\underset{(Q, f) \in \mathcal{Q}}{\oplus}(Q, f)$ is defined by

$$
(\underset{(P, e) \in \mathcal{P}}{\oplus}(P, e)) \times(\underset{(Q, f) \in \mathcal{Q}}{\oplus}(Q, f))=\underset{\substack{(P, e) \in \mathcal{P} \\(Q, f) \in \mathcal{Q}}}{\oplus}(P \times Q, e \times f) .
$$

For Assertion 2, by Proposition 10.7.2 of [8], if $F$ is a rational $p$-biset functor, the functor $F_{P}$ obtained from $F$ by the Yoneda-Dress construction at $P$ is also a rational $p$-biset functor. This applies in particular to the functor $Y=B / B_{\delta}$, so the functor $Y_{P}$ is rational. Hence, if $Q$ is any finite $p$-group and $\mathcal{B}_{Q}$ is a genetic basis of $Q$, there are mutual inverse isomorphisms

$$
Y_{P}(Q) \stackrel{\mathcal{D}_{Q}}{\underset{\mathcal{I}_{Q}}{\rightleftarrows}} \underset{S \in \mathcal{B}_{Q}}{\oplus} \partial Y_{P}\left(\bar{N}_{Q}(S)\right)
$$

where $\mathcal{I}_{Q}=\underset{S \in \mathcal{B}_{Q}}{\oplus} \operatorname{Indinf}{\overline{N_{P}}}_{\bar{P}^{(S)}}$ and $\mathcal{D}_{Q}=\underset{S \in \mathcal{B}_{Q}}{\bigoplus} f_{\mathbf{1}}^{\bar{N}_{P}(S)} \circ \operatorname{Defres} \bar{N}_{P}(S)$. Thus for any $f \in Y_{P}(Q)$

$$
f=\left(\sum_{S \in \mathcal{B}_{Q}} \operatorname{Indinf} \bar{N}_{P(S)}^{P} f_{\mathbf{1}}^{\bar{N}_{P}(S)} \operatorname{Defres}_{\bar{N}_{P}(S)}^{P}\right) \circ f .
$$


Applying this to $Q=P, \mathcal{B}_{Q}=\mathcal{B}$, and $f=\operatorname{Id}_{P}$ gives that $\mathcal{I}_{P} \circ \mathcal{D}_{P}=\operatorname{Id}_{P}$. On the other hand, by Proposition 6.4.4 and Theorem 9.6.1 of [8], for $S, T \in \mathcal{B}$, the composition

$$
f_{\mathbf{1}}^{\bar{N}_{P}(S)} \operatorname{Defres}_{\bar{N}_{P}(S)}^{P} \circ \operatorname{Indinf} \bar{N}_{P}^{P} f_{\mathbf{1}}^{\bar{N}_{P}(S)}
$$

is equal to $f_{1}^{\bar{N}_{P}(S)}$ in $B\left(\bar{N}_{P}(S), \bar{N}_{P}(S)\right)$ if $T=S$, and to 0 if $T \neq S$. It follows that $\mathcal{D}_{P} \circ \mathcal{I}_{P}$ is also equal to the identity map of the direct sum $\underset{S \in \mathcal{B}}{\bigoplus_{P}} \partial Y_{P}\left(\bar{N}_{P}(S)\right)$ in the category $\mathcal{R}_{p}$.

For Assertion 3, observe that by Lemma 2.29

$$
f_{\mathbf{1}}^{\bar{N}_{P}(S)} \operatorname{Defres}_{\bar{N}_{P}(S)} f_{1}^{P}=0
$$

if $S \cap Z(P) \neq \mathbf{1}$. Taking opposite bisets, this gives also

$$
f_{1}^{P} \operatorname{Indinf} \bar{N}_{P(S)}^{P} f_{\mathbf{1}}^{\bar{N}_{P}(S)}=0
$$

so the isomorphism of Assertion 2 restricts to an isomorphism

$$
\partial P \cong \underset{\substack{S \in \mathcal{B} \\ S \cap Z(P)=\mathbf{1}}}{\bigoplus} \partial \bar{N}_{P}(S)
$$

as the diagram

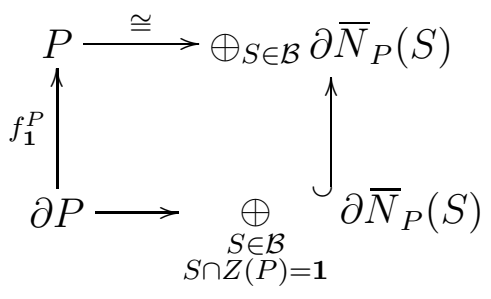

is commutative.

3.12. Corollary : Let $P$ be a finite p-group. If $Z(P)$ is non-cyclic, then $\partial P=0$ in $\mathcal{R}_{p}$.

Proof : This follows from Assertion 3 : suppose indeed that there exists a genetic subgroup $S$ of $P$ such that $S \cap Z(P) \neq 1$. Then the group $Z(P)$ maps injectively in the center of the Roquette group $\bar{N}_{P}(S)$, which is cyclic. Hence $Z(P)$ is cyclic. 


\subsection{Examples :}

1. Let $P=D_{8}$ be a dihedral group of order 8 . Let $A, B$, and $C$ be the subgroups of index 2 in $P$, and let $I$ be a non-central subgroup of order 2 in $P$. Then the set $\{P, A, B, C, I\}$ is a genetic basis of $P$, and there is an isomorphism

$$
P \cong \mathbf{1} \oplus 4 \cdot \partial C_{2}
$$

in the category $\mathcal{R}_{2}$, where $4 \cdot \partial C_{2}$ denotes the direct sum of 4 copies of $\partial C_{2}$ : indeed, for $S \in\{A, B, C, I\}$, the group $\bar{N}_{P}(S)$ is isomorphic to $C_{2}$.

2. Let $P=Q_{8}$ be a quaternion group of order 8. Let $A, B$, and $C$ be the subgroups of index 2 in $P$. Then the set $\{P, A, B, C, \mathbf{1}\}$ is a genetic basis of $P$ (such a basis is unique in this case), and there is an isomorphism

$$
P \cong \mathbf{1} \oplus 3 \cdot \partial C_{2} \oplus \partial Q_{8}
$$

in the category $\mathcal{R}_{2}$.

3. Let $P=\left(C_{p}\right)^{n}$ be an elementary abelian $p$-group of rank $n$. Then $P$ has a unique genetic basis, consisting of $P$, and all its subgroups of index $p$. Hence

$$
P \cong \mathbf{1} \oplus \frac{p^{n}-1}{p-1} \cdot \partial C_{p}
$$

in the category $\mathcal{R}_{p}$.

\section{The tensor structure}

4.1. Notation : Let $G$ and $H$ be groups. When $L$ is a subgroup of $G \times H$, set

$$
\begin{aligned}
& p_{1}(L)=\{g \in G \mid \exists h \in H,(g, h) \in L\} \\
& p_{2}(L)=\{h \in H \mid \exists g \in G,(g, h) \in L\} \\
& k_{1}(L)=\{g \in G \mid(g, 1) \in L\} \\
& k_{2}(L)=\{h \in H \mid(1, h) \in L\}
\end{aligned}
$$

Recall ([8] 2.3.18 and 2.3.21) that $k_{i}(L) \unlhd p_{i}(L)$, for $i \in\{1,2\}$, and that $\left(k_{1}(L) \times k_{2}(L)\right) \unlhd L$. Set $q(L)=L /\left(k_{1}(L) \times k_{2}(L)\right)$, and recall that there are 
canonical group isomorphisms

$$
q(L) \cong p_{1}(L) / k_{1}(L) \cong p_{2}(L) / k_{2}(L)
$$

4.2. Definition : Let $G$ and $H$ be groups. A subgroup $L$ of $(G \times H)$ will be called diagonal if

$$
L \cap(G \times \mathbf{1})=L \cap(\mathbf{1} \times H)=\mathbf{1},
$$

i.e. equivalently, if $k_{1}(L)=\mathbf{1}$ and $k_{2}(L)=\mathbf{1}$.

The subgroup $L$ will be called centrally diagonal if

$$
L \cap(Z(G) \times \mathbf{1})=L \cap(\mathbf{1} \times Z(H))=\mathbf{1},
$$

i.e. equivalently, if $k_{1}(L) \cap Z(G)=\mathbf{1}$ and $k_{2}(L) \cap Z(H)=\mathbf{1}$.

4.3. Notation : Let $G$ and $H$ be groups. When $K$ is a subgroup of $G$, and $\varphi: K \rightarrow H$ is a group homomorphism, set

$$
\vec{\Delta}_{\varphi}(K)=\{(x, \varphi(x)) \mid x \in K\} \leq G \times H
$$

and

$$
\overleftarrow{\Delta}_{\varphi}(K)=\{(\varphi(x), x) \mid x \in K\} \leq H \times G
$$

4.4. Remark : The subgroup $L$ of $(G \times H)$ is diagonal if and only if there exists a subgroup $K \leq G$ and an injective group homomorphism $\varphi: K \hookrightarrow H$ such that $L=\vec{\Delta}_{\varphi}(K)$.

4.5. Lemma : Let $P$ and $Q$ be p-groups, and let $L$ and $L^{\prime}$ be genetic subgroups of $(P \times Q)$ such that $L \widehat{=}_{P \times Q} L^{\prime}$. Then $L$ is centrally diagonal in $(P \times Q)$ if and only if $L^{\prime}$ is centrally diagonal in $(P \times Q)$.

Proof : Let $(x, y) \in(P \times Q)$ such that

$$
L^{\prime(x, y)} \cap Z_{P \times Q}(L) \leq L
$$


Since $Z(P) \times Z(Q) \leq Z_{P \times Q}(L)$, it follows that

$$
\begin{aligned}
L^{\prime} \cap(Z(P) \times 1) & =\left(L^{\prime} \cap(Z(P) \times 1)\right)^{(x, y)} \\
& =L^{\prime(x, y)} \cap(Z(P) \times 1) \\
& =L^{\prime(x, y)} \cap Z_{P \times Q}(L) \cap(Z(P) \times 1) \\
& \leq L \cap(Z(P) \times 1)=\mathbf{1} .
\end{aligned}
$$

A similar argument shows that $L^{\prime} \cap(\mathbf{1} \times Z(Q))=\mathbf{1}$.

Recall from Definition 2.8 that an axial subgroup of a finite group $G$ is a subgroup of a cyclic subgroup of maximal order of $G$ :

4.6. Theorem : Let $P$ and $Q$ be non-trivial Roquette $p$-groups, let $e_{P}$ (resp. $\left.e_{Q}\right)$ denote the exponent of $P$ (resp. of $\left.Q\right)$, and let $Z_{P}\left(\right.$ resp. $\left.Z_{Q}\right)$ denote the central subgroup of order $p$ in $P$ (resp. in $Q)$.

1. Let $L$ be a centrally diagonal genetic subgroup of $(P \times Q)$. Then $L=\vec{\Delta}_{\varphi}(H)$, where $H \leq P$ and $\varphi: H \hookrightarrow Q$ is an injective group homomorphism. Moreover, either $P \cong Q \cong Q_{8}$, and $H=P$, or $H$ is an axial subgroup of $P$ of order $\min \left(e_{P}, e_{Q}\right)$, such that $\varphi(H)$ is an axial subgroup of $Q$.

2. Conversely :

(a) if $P \cong Q \cong Q_{8}$, let $L=L_{\varphi}=\vec{\Delta}_{\varphi}(P)$, where $\varphi: P \rightarrow Q$ is a group isomorphism. Then $L$ is a centrally diagonal genetic subgroup of $(P \times Q)$, and $N_{P \times Q}(L) / L \cong C_{2}$.

(b) in all other cases, let $L=L_{\varphi}=\vec{\Delta}_{\varphi}(H)$, where $H$ is an axial subgroup of $P$ of order $\min \left(e_{P}, e_{Q}\right)$, and $\varphi: H \hookrightarrow Q$ is an injective group homomorphism such that $\varphi(H)$ is an axial subgroup of $Q$. Then $L$ is a centrally diagonal genetic subgroup of $(P \times Q)$. Moreover, the isomorphism class of the group $N_{P \times Q}(L) / L$ depends only on $P$ and $Q$.

Proof : • Observe first that the group $\left(Z_{P} \times Z_{Q}\right) L / L$ is a central subgroup of the Roquette group $N_{P \times Q}(L) / L$, hence it is cyclic. Hence $\left(Z_{P} \times Z_{Q}\right) \cap L \neq 1$. Since both $\left(Z_{P} \times \mathbf{1}\right) \cap L$ and $\left(\mathbf{1} \times Z_{Q}\right) \cap L$ are trivial, is follows that $\left(Z_{P} \times Z_{Q}\right) \cap L$ is equal to

$$
\vec{\Delta}_{\psi}\left(Z_{P}\right)=\left\{(z, \psi(z)) \mid z \in Z_{P}\right\},
$$

where $\psi: Z_{P} \stackrel{\cong}{\rightarrow} Z_{Q}$ is some group isomorphism. In particular $p_{1}(L)$ contains $Z_{P}$, and $p_{2}(L)$ contains $Z_{Q}$. 
- Let us prove now that $L$ is diagonal, i.e. that there exists a subgroup $H$ of $P$ and an injective group homomorphism $\varphi: H \hookrightarrow Q$ such that

$$
L=\vec{\Delta}_{\varphi}(H)=\{(h, \varphi(h)) \mid h \in H\} .
$$

Otherwise, at least one of the groups $k_{1}(L)$ or $k_{2}(L)$ is non trivial. But the assumption $L \cap(Z(P) \times \mathbf{1})=\mathbf{1}$ is equivalent to $L \cap\left(Z_{P} \times \mathbf{1}\right)=\mathbf{1}$, i.e. $k_{1}(L) \cap Z_{P}=\mathbf{1}$, and similarly, the assumption $L \cap(\mathbf{1} \times Z(Q))=\mathbf{1}$ is equivalent to $k_{2}(L) \cap Z_{Q}=1$. But if there exists a non trivial subgroup $X$ of $P$ such that $X \cap Z_{P}=\mathbf{1}$, then $p=2, X$ has order 2, and $P$ is dihedral or semidihedral (Lemma 2.9). So if $L$ is not diagonal, then $p=2$, and at least one of $P$ or $Q$ is dihedral or semidihedral.

Up to exchanging $P$ and $Q$, one can assume that the group $C=k_{1}(L)$ is non-trivial, hence non-central of order 2 in $P$. Set $A=p_{1}(L)$. Since $A \leq N_{P}(C)=C Z_{P}$ (Lemma 2.9) ), it follows that $q(L)=A / C$ has order 1 or 2 .

If $q(L)=\mathbf{1}$, then $A=C$, and $L=C \times D$, where $D=k_{2}(L)=p_{2}(L)$. In this case

$$
N_{P \times Q}(L) / L=\left(N_{P}(C) / C\right) \times\left(N_{Q}(D) / D\right) \cong C_{2} \times\left(N_{Q}(D) / D\right)
$$

cannot be a Roquette group, since $N_{Q}(D) / D$ is non-trivial (as $D \cap Z_{Q}=\mathbf{1}$ ).

And if $|q(L)|=2$, then $A=C Z_{P}$. If $(a, b) \in N_{P \times Q}(L)$, then in particular $a \in N_{P}(A, C)=A$. Thus $N_{P \times Q}(L) \leq A \times Q$. Now the group $N_{P}(A)$ is a proper subgroup of $P$, since $A$ is elementary abelian of rank 2 , and $P$ is a Roquette group. Choose $x \in P-N_{P}(A)$, whence $A^{x} \cap A=Z_{P}$. If $(a, b) \in L^{(x, 1)} \cap(A \times Q)$, then $a \in A^{x} \cap A=Z_{P}$, hence $(a, b)={ }^{(x, 1)}(a, b) \in L$. Thus $L^{(x, 1)} \cap(A \times Q) \leq L$, and

$$
\begin{aligned}
L^{(x, 1)} \cap Z_{P \times Q}(L) & \leq L^{(x, 1)} \cap N_{P \times Q}(L) \\
& \leq L^{(x, 1)} \cap(A \times Q) \\
& \leq L
\end{aligned}
$$

but $L^{(x, 1)} \neq L$, since $A^{x} \neq A$. It follows that $L$ is not expansive in $(P \times Q)$, hence $L$ is not a genetic subgroup of $(P \times Q)$.

- Hence $L$ is diagonal in $(P \times Q)$, i.e.

$$
L=\vec{\Delta}_{\varphi}(H)=\{(h, \varphi(h)) \mid h \in H\}
$$

for some subgroup $H \geq Z_{P}$ of $P$ and some $\varphi: H \hookrightarrow Q$, such that $\varphi(H) \geq Z_{Q}$. Then

$$
N_{P \times Q}(L)=\left\{(x, y) \in N_{P}(H) \times N_{Q}(\varphi(H)) \mid \forall h \in H, \quad \varphi\left({ }^{x} h\right)={ }^{y} \varphi(h)\right\} .
$$


The unique central subgroup of order $p$ of the Roquette group $N_{P \times Q}(L) / L$ is equal to $Z / L$, where

$$
Z=\left(Z_{P} \times \mathbf{1}\right) L=\left(\mathbf{1} \times Z_{Q}\right) L .
$$

For any $(x, y) \in(P \times Q)$, saying that $L^{(x, y)} \cap Z_{P \times Q}(L)$ is contained in $L$ is equivalent to saying that the group $I=L^{(x, y)} \cap Z$ is contained in $L$. In particular, for $y=1$

$$
\begin{aligned}
I & =L^{(x, 1)} \cap\left(Z_{P} \times \mathbf{1}\right) L \\
& =\left\{\left(h^{x}, \varphi(h)\right) \mid h \in H, \exists z \in Z_{P}, \exists h^{\prime} \in H,\left(h^{x}, \varphi(h)\right)=\left(z h^{\prime}, \varphi\left(h^{\prime}\right)\right)\right\} \\
& =\left\{\left(h^{x}, \varphi(h)\right) \mid h \in H, h^{-1} h^{x} \in Z_{P}\right\},
\end{aligned}
$$

since $\varphi(h)=\varphi\left(h^{\prime}\right)$ implies $h=h^{\prime}$, and since $Z_{P}$ is central in $P$. Denoting by $[h, x]=h^{-1} h^{x}$ the commutator of $h$ and $x$, it follows that $I \leq L$ if and only if

$$
\forall h \in H, \quad[h, x] \in Z_{P} \Longrightarrow h^{x} \in H,\left(h^{x}, \varphi(h)\right)=\left(h^{x}, \varphi\left(h^{x}\right)\right) .
$$

In other words $[h, x] \in Z_{P}$ implies $h^{x}=h$. Thus $I \leq L$ if and only if

$$
\forall h \in H, \quad[h, x] \in Z_{P} \Longrightarrow[h, x]=1 .
$$

Equivalently $[H, x] \cap Z_{P}=\{1\}$, where $[H, x]$ denotes the set of commutators $[h, x]$, for $h \in H$.

Since $L=\vec{\Delta}_{\varphi}(H)$ is expansive in $(P \times Q)$, it follows that

$$
[H, x] \cap Z_{P}=\{1\} \Longrightarrow L^{(x, 1)}=L .
$$

Now $(x, 1)$ normalizes $L$ if and only if $x \in C_{P}(H)$, i.e. if $[H, x]=\{1\}$. Hence

$$
[H, x] \cap Z_{P}=\{1\} \Longrightarrow[H, x]=\{1\} \text {. }
$$

- Let us show now that unless $H=P \cong Q \cong Q_{8}$, the group $H$ is an axial subgroup of $P$, and the subgroup $\varphi(H)$ is an axial subgroup of $Q$.

Let $X$ be a cyclic subgroup of $P$ of order $e_{P}$, let $x$ be a generator of $X$, and suppose that $H \not X X$. Then in particular $P$ is not cyclic, so $p=2$, the group $X$ is a (normal) subgroup of index 2 of $P$ (Lemma 2.9), and $X$ is equal to its centralizer in $P$. Moreover $|H: H \cap X|=2$ since $H \cdot X=P$. The set $[H, x]$ is equal to $\left\{1, x^{2}\right\}$ if $P$ is cyclic or generalized quaternion, or to $\left\{1, x^{2+2^{n-2}}\right\}$ if $P$ is semidihedral : indeed, the image of $H$ in the group of automorphisms of $X$ has order 2 , as $H \cap X$ centralizes $X$, and $H$ does not. Since $Z_{P}$ is generated by $x^{2^{n-2}}$, it follows that $[H, x] \cap Z_{P}=\{1\}$ if $n \geq 4$, 
i.e. if $|P| \geq 16$. But $[H, x] \neq\{1\}$, hence $L$ is not expansive in $(P \times Q)$, if $P \geq 16$.

So if $H \not \leq X$, then $p=2$, and $P$ is non-cyclic, of order at most 8 . Hence $P \cong Q_{8}$. If $H \neq P$, then $H$ is cyclic, and $H \not X X$. Thus $|H|=4=e_{P}$, and in particular $H$ is an axis of $P$. Since $H$ embeds into $Q$, it follows that $|H|=\min \left(e_{P}, e_{Q}\right)$. The same argument applied to $\varphi(H)$ shows that $\varphi(H)$ is an axial subgroup of $Q$, as claimed.

In this case moreover, the group $Q$ cannot be isomorphic to $Q_{8}$ : indeed otherwise, one can assume that $P=Q$ and $L=\Delta(H)$ is the diagonal embedding. Then

$$
N_{P \times P}(L)=\left\{(a, b) \mid a^{-1} b \in H\right\} .
$$

The group $N_{P \times P}(L) / L$ has order 8 , generated by the cyclic subgroup

$$
C=\{(a, 1) L \mid a \in H\}
$$

of index 2 , and the involution $(b, b) L$, where $b \in P-H$. Hence $N_{P \times P}(L) / L \cong$ $D_{8}$ is not a Roquette group, and $L$ is not a genetic subgroup of $(P \times P)$.

If $H$ is non-cyclic, then $H=P$, and the same argument applied to $\varphi(H)$ shows that $Q \cong Q_{8}$. And indeed $\vec{\Delta}_{\varphi}(P)$ is a genetic subgroup of $P \times Q$ : this follows from Example 2.17, since the map $(x, y) \mapsto\left(x, \varphi^{-1}(y)\right)$ is a group isomorphism from $(P \times Q)$ to $(P \times P)$, sending $\vec{\Delta}_{\varphi}(P)$ to $\Delta(P)$. Moreover $[P, P] \leq Z(P)$, and $Z(P)$ has order 2. In particular

$$
N_{P \times Q}(L) / L \cong C_{2}
$$

does not depend on $\varphi$, up to isomorphism. This proves part a) of Assertion 2.

- In the remaining cases $L=\vec{\Delta}_{\varphi}(H)$, where $H$ is a non-trivial axial subgroup of $P$, and $\varphi: H \hookrightarrow Q$ is such that $\varphi(H)$ is an axial subgroup of $Q$. In particular $H$ is cyclic, and non-trivial. As $H \cong \varphi(H) \leq Q$, it follows that $|H| \leq \min \left(e_{P}, e_{Q}\right)$.

Let $C_{e_{P}}$ be an axis of $P$ containing $H$, and $C_{e_{Q}}$ be an axis of $Q$ containing $\varphi(Q)$. Then $\left(C_{e_{P}} \times C_{e_{Q}}\right) / \Delta_{\varphi}(H)$ is an abelian normal subgroup of the Roquette group $N_{P \times Q}(L) / L$, hence it is cyclic. Thus $L=\Delta_{\varphi}(H)$ is not contained in the Frattini subgroup $\left(C_{e_{P} / p} \times C_{e_{Q} / p}\right)$ of $\left(C_{e_{P}} \times C_{e_{Q}}\right)$. In particular $p_{1}(L)=C_{e_{Q}}$, or $p_{2}(L)=C_{e_{Q}}$. In other words $H=C_{e_{P}}$, or $\varphi(H)=C_{e_{Q}}$, hence $|H|=\min \left(e_{P}, e_{Q}\right)$. This completes the proof of Assertion 1.

- Now assume that at least one of the groups $P$ or $Q$ is not isomorphic to $Q_{8}$. Assume also that $e_{P} \leq e_{Q}$, and let $H$ be an axis of $P$ : then $H$ is unique if 
$P \neq Q_{8}$, and there are three possibilities for $H$ if $P \cong Q_{8}$ (Lemma 2.9). In any case $H \unlhd P$. Let $K$ denote an axial subgroup of $Q$ of order $e_{P}$. Such a group is unique, except if $p=2, e_{P}=4$, and $Q \cong Q_{8}$ (thus $P \cong C_{4}$ as $P$ has exponent 4 , and is not isomorphic to $\left.Q_{8}\right)$. In any case $K \unlhd Q$.

Let $\varphi: H \stackrel{\cong}{\rightarrow} K$ be any group isomorphism, and set $L_{\varphi}=\vec{\Delta}_{\varphi}(H) \leq$ $(P \times Q)$. Then $L_{\varphi}$ is obviously centrally diagonal, and

$$
N_{P \times Q}\left(L_{\varphi}\right)=\left\{(a, b) \in(P \times Q) \mid \forall h \in H, \quad \varphi\left({ }^{a} h\right)={ }^{b} \varphi(h)\right\} .
$$

Since $H$ is cyclic of order $e_{P}$, the map

$$
\pi_{H}: r \in\left(\mathbb{Z} / e_{P} \mathbb{Z}\right)^{\times} \mapsto\left(x \mapsto x^{r}\right) \in \operatorname{Aut}(H)
$$

is a canonical group isomorphism. Similarly, the map

$$
\pi_{K}: r \in\left(\mathbb{Z} / e_{P} \mathbb{Z}\right)^{\times} \mapsto\left(x \mapsto x^{r}\right) \in \operatorname{Aut}(K)
$$

is a canonical group isomorphism.

Let $\alpha: P \rightarrow \operatorname{Aut}(H) \stackrel{\pi_{H}^{-1}}{\longrightarrow}\left(\mathbb{Z} / e_{P} \mathbb{Z}\right)^{\times}$denote the group homomorphism obtained from the action of $P$ on its normal subgroup $H$ by conjugation, and $\beta: Q \rightarrow \operatorname{Aut}(K) \stackrel{\pi_{K}^{-1}}{\longrightarrow}\left(\mathbb{Z} / e_{P} \mathbb{Z}\right)^{\times}$denote the group homomorphism obtained from the action of $Q$ on its normal subgroup $K$. Then

$$
N_{P \times Q}\left(L_{\varphi}\right)=\{(a, b) \in(P \times Q) \mid \alpha(a)=\beta(b)\} .
$$

Now the group $\left(\mathbb{Z} / e_{P} \mathbb{Z}\right)^{\times}$is abelian. The map

$$
\Theta:(a, b) \in(P \times Q) \mapsto \beta(b)^{-1} \cdot \alpha(a) \in\left(\mathbb{Z} / e_{P} \mathbb{Z}\right)^{\times}
$$

is a group homomorphism, and $N_{P \times Q}\left(L_{\varphi}\right)=\operatorname{Ker} \Theta$. In particular, it is a normal subgroup of $(P \times Q)$, which does not depend on $\varphi$, once $H$ and $K=\varphi(H)$ are fixed. In particular $L_{\varphi}$ is an expansive subgroup of $(P \times Q)$, by Example 2.14. Moreover setting $I_{P, Q}=\operatorname{Im}(\alpha) \cap \operatorname{Im}(\beta)$, there is an exact sequence

$$
\mathbf{1} \rightarrow C_{P}(H) \times C_{Q}(K) \rightarrow N_{P \times Q}\left(L_{\varphi}\right) \stackrel{\Psi}{\longrightarrow} I_{P, Q} \rightarrow \mathbf{1},
$$

where $\Psi(a, b)=\alpha(a)=\beta(b)$, for $(a, b) \in N_{P \times Q}\left(L_{\varphi}\right)$.

$\S$. Suppose first that $e_{P}=p$, i.e. that $P \cong C_{p}$. In this case $H=Z_{P}=P$, and $K=Z_{Q}$, so $L_{\varphi}$ is central in $(P \times Q)$. Moreover

$$
N_{P \times Q}\left(L_{\varphi}\right) / L_{\varphi}=(P \times Q) / L_{\varphi}=(P \times Q) / \vec{\Delta}_{\varphi}(P) \cong Q
$$


is a Roquette group, independent of $\varphi$, up to isomorphism. In particular $L_{\varphi}$ is a genetic subgroup of $(P \times Q)$.

$\S$. Assume from now on that $e_{P} \geq p^{2}$. Then $C_{P}(H) \cong C_{e_{P}}$, and $C_{Q}(K) \cong$ $C_{e_{Q}}$, by Lemma 2.9.

$\S \S$. If $p>2$, then $P$ and $Q$ are cyclic, hence $H=P$, and

$$
N_{P \times Q}\left(L_{\varphi}\right) / L_{\varphi} \cong(P \times Q) / \vec{\Delta}_{\varphi}(P) \cong Q
$$

as above. It is a Roquette group, independent of $\varphi$, up to isomorphism. In particular $L_{\varphi}$ is genetic in $(P \times Q)$.

$\S \S$. Assume now that $p=2$. The image of $\alpha$ has order $\left|P: C_{P}(H)\right|$, which is equal to 1 if $P$ is cyclic, and to 2 otherwise. Similarly, the image of $\beta$ has order $\left|Q: C_{Q}(K)\right|$, which is equal to 1 if $K$ is central in $Q$, i.e. if $Q$ is cyclic (since $|K|=e_{P} \geq 4$ by assumption), and to 2 otherwise. Set $I_{P, Q}=\operatorname{Im}(\alpha) \cap \operatorname{Im}(\beta)$. Then $I_{P, Q}$ has order 1 or 2 , and there is an exact sequence

$$
1 \rightarrow C_{e_{P}} \times C_{e_{Q}} \rightarrow N_{P \times Q}\left(L_{\varphi}\right) \rightarrow I_{P, Q} \rightarrow 1
$$

Note that $I_{P, Q}$ does not depend on $\varphi: H \stackrel{\cong}{\rightrightarrows} K:$ more precisely

$$
\operatorname{Im}(\alpha)=\left\{\begin{array}{cl}
\{1\} & \text { if } P \text { is cyclic, } \\
\{1,-1\} & \text { if } P \text { is dihedral or generalized quaternion, } \\
\left\{1, e_{P} / 2-1\right\} & \text { if } P \text { is semidihedral. }
\end{array}\right.
$$

So $\operatorname{Im}(\alpha)$ only depends on the type of $P$.

Similarly

$$
\operatorname{Im}(\beta)=\left\{\begin{array}{cl}
\{1\} & \text { if } Q \text { is cyclic, } \\
\{1,-1\} & \text { if } Q \text { is dihedral or generalized quaternion, } \\
\left\{1, e_{Q} / 2-1\right\} & \text { if } Q \text { is semidihedral. }
\end{array}\right.
$$

Moreover if $Q$ is semidihedral and if $e_{Q}>e_{P}$, then $e_{Q} / 2-1 \equiv-1\left(\bmod \cdot e_{P}\right)$, hence $\operatorname{Im}(\beta)=\{1,-1\}$. In other words, the group $I_{P, Q}$ is trivial in one of the following cases :

- $P$ or $Q$ is cyclic,

- $P$ is dihedral or generalized quaternion, $Q$ is semidihedral, and $e_{P}=e_{Q}$, (i.e. equivalently $|P|=|Q|$ ),

- $P$ is semidihedral, and $Q$ is dihedral or generalized quaternion,

- $P$ and $Q$ are semidihedral, and $e_{P}<e_{Q}$ (i.e. equivalently $|P|<|Q|$ ), 
and the group $I_{P, Q}$ has order 2 in all other cases, i.e. in one of the following cases :

- $P$ and $Q$ are dihedral or generalized quaternion,

- $P$ is dihedral or generalized quaternion, $Q$ is semidihedral, and $|Q|>|P|$,

- $P$ and $Q$ are semidihedral, and $P \cong Q$.

As $L_{\varphi} \leq C_{e_{P}} \times C_{e_{Q}}$, the exact sequence 4.14 yields the exact sequence

$$
1 \rightarrow\left(C_{e_{P}} \times C_{e_{Q}}\right) / L_{\varphi} \rightarrow N_{P \times Q}\left(L_{\varphi}\right) / L_{\varphi} \rightarrow I_{P, Q} \rightarrow 1
$$

Case 1: If $I_{P, Q}$ is trivial, then $N_{P \times Q}\left(L_{\varphi}\right) \cong C_{e_{P}} \times C_{e_{Q}}$, and

$$
N_{P \times Q}\left(L_{\varphi}\right) / L_{\varphi} \cong C_{e_{Q}},
$$

which is a Roquette group, independent of $\varphi$, up to isomorphism. In particular $L_{\varphi}$ is a genetic subgroup of $(P \times Q)$.

Case 2: Suppose now that $I_{P, Q}$ has order 2, i.e. that $\operatorname{Im}(\alpha)=\operatorname{Im}(\beta)=\{1, \epsilon\}$, where $\epsilon$ is either -1 or $e_{Q} / 2-1$ (in the case where $P$ and $Q$ are semidihedral and isomorphic). One can choose an element $u \in P$, of order 2 if $P$ is dihedral or semidihedral, and of order 4 if $P$ is generalized quaternion, such that $\alpha(u)=\epsilon$. Similarly, one can choose an element $v \in Q$, of order 2 if $Q$ is dihedral or semidihedral, and of order 4 if $Q$ is generalized quaternion, such that $\beta(v)=\epsilon$. These choices imply that $(u, v) \in N_{P \times Q}\left(L_{\varphi}\right)$.

In the exact sequence 4.15

$$
1 \rightarrow\left(C_{e_{P}} \times C_{e_{Q}}\right) / L_{\varphi} \rightarrow N_{P \times Q}\left(L_{\varphi}\right) / L_{\varphi} \rightarrow\{1, \epsilon\} \rightarrow 1,
$$

the group $C=\left(C_{e_{P}} \times C_{e_{Q}}\right) / L_{\varphi}$ is cyclic, isomorphic to $C_{e_{Q}}$. The element $\pi=(u, v) L_{\varphi}$ of $N_{P \times Q}\left(L_{\varphi}\right) / L_{\varphi}$ acts on $C$ in the same way that $v$ acts on the subgroup $C_{e_{Q}}$ of $Q$, namely by inversion if $Q$ is dihedral or generalized quaternion, and by raising elements to the power $e_{Q} / 2-1$ if $Q$ is semidihedral. Finally $\pi^{2}=\left(u^{2}, v^{2}\right) L_{\varphi}=L_{\varphi}$ if none of $P$ and $Q$ are generalized quaternion. If $P$ is generalized quaternion and $Q$ is not, then $\pi^{2}=\left(z_{P}, 1\right) L_{\varphi} \in C-\{1\}$, where $z_{P}$ is a generator of $Z_{P}$. Similarly, if $Q$ is generalized quaternion and $P$ is not, then $\pi^{2}=\left(1, z_{Q}\right) L_{\varphi} \in C-\{1\}$, where $z_{Q}$ is a generator of $Z_{Q}$. In these two cases $\pi^{4}=(1,1) L_{\varphi}=L_{\varphi}$, so $\pi$ has order 4 in $N_{P \times Q}\left(L_{\varphi}\right) / L_{\varphi}$. And finally, if both $P$ and $Q$ are generalized quaternion, then $\pi^{2}=\left(z_{P}, z_{Q}\right) L_{\varphi}=L_{\varphi}$, since $\varphi\left(Z_{P}\right)=Z_{Q}$.

It follows that the group $N_{P \times Q}\left(L_{\varphi}\right) / L_{\varphi}$ has order $2 e_{Q}=|Q|$, and that it is : 
- dihedral if $P$ and $Q$ are both dihedral, or both generalized quaternion,

- generalized quaternion if one of $P, Q$ is generalized quaternion, and the other is dihedral,

- semidihedral if $Q$ is semidihedral.

So $N_{P \times Q}\left(L_{\varphi}\right) / L_{\varphi}$ is a Roquette group, independent of $\varphi$, up to isomorphism. In particular, it follows that $L_{\varphi}$ is a genetic subgroup of $(P \times Q)$. This completes the proof of Theorem 4.6.

\subsection{Notation :}

- Let $P$ and $Q$ be Roquette p-groups. If $P$ and $Q$ are non-trivial, set $P \diamond Q=N_{P \times Q}(L) / L$, where $L$ is a centrally diagonal genetic subgroup of $(P \times Q)$. Set moreover $\mathbf{1} \diamond P=P \diamond \mathbf{1}=P$.

- Let $P$ and $Q$ be Roquette $p$-groups. If $P$ and $Q$ are non-trivial, let $\nu_{P, Q}$ denote the number of equivalence classes of centrally diagonal genetic subgroups of $(P \times Q)$ for the relation $\widehat{C}_{P \times Q}$. Set moreover $\nu_{1, P}=$ $\nu_{P, \mathbf{1}}=1$.

4.19. Remark : if $P=\mathbf{1}$, and $Q$ is a Roquette $p$-group, then $P \times Q \cong Q$, and the centrally diagonal genetic subgroups of $P \times Q$ are the subgroups $\mathbf{1} \times R$, where $R$ is a genetic subgroup of $Q$ such that $R \cap Z(Q)=\mathbf{1}$. The only such subgroup is $R=\mathbf{1}$, so $N_{Q}(R) / R \cong Q \cong N_{P \times Q}(\mathbf{1} \times R) /(\mathbf{1} \times R)$. Hence the above definition of $P \diamond Q$ and $\nu_{P, Q}$ is consistent, in the case $P=\mathbf{1}$.

4.20. Theorem : Let $P$ and $Q$ be Roquette p-groups, of exponents $e_{P}$ and $e_{Q}$, respectively. Suppose $e_{P} \leq e_{Q}$, and set $q=|Q|$. Then

$$
P \diamond Q \cong \begin{cases}Q & \text { if } P=\mathbf{1} \text { or } P \cong C_{p}, \\ C_{2} \quad \text { if } P \cong Q \cong Q_{8}, \\ D_{q} \quad \text { if } q \geq 16 \text { and } P \text { and } Q \text { are both dihedral, } \\ \quad \text { or both generalized quaternion, } \\ Q_{q} \quad \text { if one of } P, Q \text { is dihedral, } \\ \quad \text { and the other one is generalized quaternion, } \\ S D_{q} \quad \text { if } Q \text { is semidihedral, and } \\ \quad-\text { either } P \text { is dihedral or generalized quaternion, } \\ \quad \text { and }|P|<|Q|, \\ \quad \text { or } P \cong Q \\ C_{e_{Q}} \quad \text { otherwise. }\end{cases}
$$


Proof : The case $P=1$ is trivial, the case $P \cong C_{p}$ follows from 4.12, the case $P \cong Q \cong Q_{8}$ follows from 4.10, the three next cases in the list follow from 4.17, and the last case follows from 4.13 and 4.16 .

4.21. Theorem : Let $P$ and $Q$ be Roquette p-groups, of exponent $e_{P}$ and $e_{Q}$, respectively, and let $m=\min \left(e_{P}, e_{Q}\right)$.

1. If $p=2$ and and one of the groups $P$ or $Q$ is isomorphic to $Q_{8}$, then then $L \widehat{-}_{P \times Q} L^{\prime}$ for any centrally diagonal genetic subgroups $L$ and $L^{\prime}$ of $P \times Q$. In other words $\nu_{P, Q}=1$.

2. In all other cases, if $L$ and $L^{\prime}$ are centrally diagonal genetic subgroups of $P \times Q$, then $L \widehat{=}_{P \times Q} L^{\prime}$ if and only if $L$ and $L^{\prime}$ are conjugate in $P \times Q$. In particular $\nu_{P, Q}=\phi(m) m \frac{|P \diamond Q|}{|P||Q|}$, where $\phi$ is the Euler function.

Proof : Assume $e_{P} \leq e_{Q}$, without loss of generality.

1) If $P=\mathbf{1}$, then $\nu_{P, Q}=1$, and $P \diamond Q=Q$ by definition, and $e_{P}=1$, so there is nothing to prove.

2) If $p=2$ and $P \cong Q \cong Q_{8}$, then by Theorem 4.6, a genetic centrally diagonal subgroup $L$ of $P \times Q$ is of the form $L_{\varphi}=\vec{\Delta}_{\varphi}(P)$, where $\varphi: P \rightarrow Q$ is some group isomorphism. Moreover $N_{P \times Q}(L) / L \cong C_{2}$, so $P \diamond Q \cong C_{2}$.

Now let $\varphi, \psi: P \rightarrow Q$ be two group isomorphisms. Then $L_{\varphi} \widehat{=}_{P \times Q} L_{\psi}$ if and only if there exists $(x, y) \in(P \times Q)$ such that

$$
\begin{aligned}
L_{\varphi}^{(x, y)} \cap Z_{P \times Q}\left(L_{\psi}\right) & \leq L_{\psi} \\
{ }^{(x, y)} L_{\psi} \cap Z_{P \times Q}\left(L_{\varphi}\right) & \leq L_{\varphi} .
\end{aligned}
$$

These conditions depend only on the double coset $N_{P \times Q}\left(L_{\varphi}\right)(x, y) N_{P \times Q}\left(L_{\psi}\right)$, which admits a representative of the form $(u, 1)$.

Now the condition $L_{\varphi}^{(u, 1)} \cap Z_{P \times Q}\left(L_{\psi}\right) \leq L_{\psi}$ is equivalent to

$$
\forall h \in P, \varphi(h)^{-1} \psi\left(h^{u}\right) \in Z_{Q} \Longrightarrow \varphi(h)^{-1} \psi\left(h^{u}\right)=1 \text {, }
$$

and similarly, the condition ${ }^{(u, 1)} L_{\psi} \cap Z_{P \times Q}\left(L_{\varphi}\right) \leq L_{\varphi}$ is equivalent to

$$
\forall h \in P, \quad \psi(h)^{-1} \varphi\left({ }^{u} h\right) \in Z_{Q} \Longrightarrow \psi(h)^{-1} \varphi\left({ }^{u} h\right)=1 \text {. }
$$


Applying $\psi^{-1}$ to the first condition and $\varphi^{-1}$ to the second one, and setting $\theta=\psi^{-1} \varphi$, these two conditions become

$$
\begin{aligned}
\forall h \in P, \quad \theta(h)^{-1} h^{u} \in Z_{P} & \Longrightarrow \theta(h)=h^{u} \\
\forall h \in P, \quad \theta^{-1}(h)^{-1 u} h \in Z_{P} & \Longrightarrow \theta^{-1}(h)={ }^{u} h .
\end{aligned}
$$

Since $h^{u} h^{-1} \in[P, P]=Z_{P}$, there are equivalences

$$
\begin{aligned}
& \theta(h)^{-1} h^{u} \in Z_{P} \Longleftrightarrow \theta(h)^{-1} h \in Z_{P} \quad \Longleftrightarrow h^{-1} \theta(h) \in Z_{P}, \\
& \theta^{-1}(h)^{-1 u} h \in Z_{P} \Longleftrightarrow \theta^{-1}(h)^{-1} h \in Z_{P} \Longleftrightarrow h^{-1} \theta(h) \in Z_{P} \text {. }
\end{aligned}
$$

Hence, in order to prove that $L_{\varphi} \widehat{=}_{P \times Q} L_{\psi}$ for any $\varphi, \psi: P \stackrel{\rightrightarrows}{\rightarrow} Q$, it is enough to prove that

$$
\forall \theta \in \operatorname{Aut}(P), \exists u \in P, \forall h \in P, \quad \theta(h) h^{-1} \in Z_{P} \Longrightarrow\left\{\begin{array}{l}
\theta(h)=h^{u} \\
\theta^{-1}(h)={ }^{u} h
\end{array} .\right.
$$

If $h$ has order 1 or 2 , then $\theta(h)=h=h^{u}$ for any $u \in P$, hence the conditions $\theta(h)=h^{u}$ and $\theta^{-1}(h)={ }^{u} h$ only have to be checked for $|h|=4$. Now the group $\operatorname{Aut}(P)$ permutes the three cyclic subgroups of order 4 of $P$, and this gives an exact sequence

$$
1 \rightarrow \operatorname{Inn}(\mathrm{P}) \rightarrow \operatorname{Aut}(\mathrm{P}) \rightarrow \mathrm{S}_{3} \rightarrow 1
$$

where $S_{3}$ is the symmetric group on three symbols. Saying that $\theta(h) h^{-1} \in Z_{P}$ is equivalent to saying that $\theta(\langle h\rangle)=\langle h\rangle$. Hence, either $\theta$ stabilizes the three subgroups of order 4 of $P$, and in this case $\theta$ is inner, hence there exists $u \in P$ such that $\theta(h)=h^{u}$, for any $h \in P$, hence $\theta^{-1}(h)={ }^{u} h$, for any $h \in P$.

Or there exists a unique subgroup $C$ of order 4 of $P$ such that $\theta(C)=C$. Then either $\theta(h)=h$ for any $h \in C$, or $\theta(h)=h^{-1}$, for any $h \in C$. In the first case, take $u=1$, and in the second case take $u \in P-\langle h>$, and then $\theta(h)=h^{u}$ for any $h \in C$, hence $h=\theta^{-1}(h)^{u}$ for $h \in C$, since $C=\theta(C)$. Hence 4.22 holds. This completes the proof in this case.

3) If $P \cong Q_{8}$ and $Q \nsubseteq Q_{8}$, then a centrally diagonal genetic subgroup of $(P \times Q)$ is of the form $L=\vec{\Delta}_{\varphi}(H)$, where $H$ is one of the 3 subgroups of order 4 of $P$, and $\varphi$ is some isomorphism from $H$ to the unique axial subgroup $K$ of order 4 of $Q$. Moreover $Z_{P \times Q}(L)=L\left(\mathbf{1} \times Z_{Q}\right)$.

Let $H$ and $H^{\prime}$ be subgroups of order 4 of $P$. Let $\varphi: H \rightarrow K$ and $\varphi^{\prime}: H^{\prime} \rightarrow K$ be group isomorphisms, and set $L=\vec{\Delta}(\varphi)$ and $L^{\prime}=\vec{\Delta}_{\varphi^{\prime}}\left(H^{\prime}\right)$. Suppose first that $H \neq H^{\prime}$, and let $(a, b) \in L^{\prime} \cap Z_{P \times Q}(L)=L\left(\mathbf{1} \times Z_{Q}\right)$. It means that $a \in H^{\prime} \cap H=Z_{P}$, and that there exists $z \in Z_{Q}$ such that $\varphi^{\prime}(a)=$ 
$\varphi(a) z$. But the restrictions of $\varphi$ and $\varphi^{\prime}$ to $Z_{P}$ are equal, so $\varphi^{\prime}(a)=\varphi(a)$, hence $z=1$. It follows that $L^{\prime} \cap Z_{P \times Q}(L) \leq L$, hence $L \cap Z_{P \times Q}\left(L^{\prime}\right) \leq L^{\prime}$ by symmetry, so $L \widehat{=}_{P \times Q} L^{\prime}$ in this case.

Now if $H=H^{\prime}$, choose a subgroup $H^{\prime \prime}$ of order 4 in $P$, different from $H$, and a group isomorphism $\varphi^{\prime \prime}: H^{\prime \prime} \rightarrow K$. Set $L^{\prime \prime}=\vec{\Delta}_{\varphi^{\prime \prime}}\left(H^{\prime \prime}\right)$. Then $L \widehat{=}_{P \times Q} L^{\prime \prime} \widehat{=}_{P \times Q} L^{\prime}$, by the previous argument, thus $L \widehat{ }_{P \times Q} L^{\prime}$.

Hence $\nu_{P, Q}=1$ in this case, as was to be shown.

4) In the case there are several choices for $K$, i.e. if $Q \cong Q_{8}$ and $K$ has order $4=\min \left(e_{P}, e_{Q}\right)$, it follows that $P \cong C_{4}$, since $P \nsubseteq Q_{8}$. In this case, we can exchange $P$ and $Q$, and use the previous argument. Hence $\nu_{P, Q}=1$ in this case as well.

5) In all other cases, by Theorem 4.6, a centrally diagonal genetic subgroup $L$ of $(P \times Q)$ is of the form $\vec{\Delta}_{\varphi}(H)$, where $H \leq P$ is the unique axis of $P$, and $\varphi: H \hookrightarrow Q$ is a group isomorphism to the unique axial subgroup $K$ of order $e_{P}$ of $Q$. The normalizer of $L$ in $P \times Q$ does not depend on $\varphi$, by 4.11, so it does not depend on $L$, since $H$ and $K$ are also unique.

Let $L$ and $L^{\prime}$ be two such centrally diagonal genetic subgroups of $P \times Q$. Then $N_{P \times Q}(L)=N_{P \times Q}\left(L^{\prime}\right)$, thus $L \widehat{=}_{P \times Q} L^{\prime}$ if and only if $L$ and $L^{\prime}$ are conjugate in $(P \times Q)$, by Lemma 2.20. Moreover, it follows from the definition of $P \diamond Q$ that

$$
\left|N_{P \times Q}(L)=\right| L|| P \diamond Q\left|=e_{P}\right| P \diamond Q \mid,
$$

so the conjucacy class of $L$ in $(P \times Q)$ has cardinality $\frac{|P||Q|}{e_{P}|P \diamond Q|}$. Since there are $\phi\left(e_{P}\right)$ possible choices for the isomorphism $\varphi: H \rightarrow K$, i.e. $\phi\left(e_{P}\right)$ centrally diagonal subgroups of $(P \times Q)$, it follows that

$$
\nu_{P, Q}=\phi\left(e_{P}\right) e_{P} \frac{|P \diamond Q|}{|P||Q|},
$$

as was to be shown.

4.23. Remark : Suppose that $P \cong Q_{8}$ and $Q \nsubseteq Q_{8}$. Then $|P \diamond Q|=|Q|$, by Theorem 4.20, Hence

$$
\phi\left(e_{P}\right) e_{P} \frac{|P \diamond Q|}{|P||Q|}=2 \times 4 \times \frac{|Q|}{8|Q|}=1=\nu_{P, Q}
$$

so the formula for $\nu_{P, Q}$ holds in this case. The only case where $\nu_{P, Q}$ is not equal to $\phi(m) m \frac{|P \diamond Q|}{|P||Q|}$ (where $\left.m=\min \left(e_{P}, e_{Q}\right)\right)$ is the case $P \cong Q \cong Q_{8}$ : 
in this case $\nu_{P, Q}=1$, but

$$
\phi(m) m \frac{|P \diamond Q|}{|P||Q|}=2 \times 4 \times \frac{2}{8 \times 8}=\frac{1}{4} .
$$

4.24. Corollary : Let $P$ and $Q$ be Roquette p-groups. Then, in the category $\mathcal{R}_{p}$

$$
\partial P \times \partial Q \cong \nu_{P, Q} \cdot \partial(P \diamond Q)
$$

In other words, if $P$ has exponent $e_{P}$, if $Q$ has order $q$ and exponent $e_{Q}$, and if $e_{P} \leq e_{Q}$ :

$$
\partial P \times \partial Q= \begin{cases}\partial Q & \text { if } P=\mathbf{1} \text { or } P \cong C_{2}, \\ \partial C_{2} & \text { if } P \cong Q \cong Q_{8}, \\ \frac{\phi\left(e_{P}\right)}{2} \cdot \partial D_{q} & \text { if } q \geq 16 \text { and } P \text { and } Q \text { are both dihedral, } \\ \frac{\phi\left(e_{P}\right)}{2} \cdot \partial Q_{q} & \text { or both generalized quaternion, } \\ \frac{\phi\left(e_{P}\right)}{2} \cdot \partial S D_{q} & \text { if one of } P, Q \text { is generalized quaternion, } \\ & \text { and the other one is dihedral, } \\ & \text { - ither } P \text { is dihedral }, \text { and } \text { or } P \cong Q, \\ \frac{\phi\left(e_{P}\right) e_{P} e_{Q}}{|P||Q|} \cdot \partial C_{e_{Q}} & \text { otherwise } .\end{cases}
$$

Proof : Let $\mathcal{B}$ be a genetic basis of the group $R=P \times Q$. In the category $\mathcal{R}_{p}$, the product $\partial P \times \partial Q$ is equal to $\left(P \times Q, f_{\mathbf{1}}^{P} \times f_{\mathbf{1}}^{Q}\right)$, and it is a summand of $R$. By Theorem 3.11, there are mutual inverse isomorphisms

$$
R \stackrel{\mathcal{D}}{\underset{\mathcal{I}}{\rightleftarrows}} \underset{S \in \mathcal{B}}{\oplus} \partial \bar{N}_{R}(S)
$$

where $\mathcal{I}$ is the direct sum of the maps $\operatorname{Indinf} \frac{R}{\bar{N}_{R}(S)}$, and $\mathcal{D}$ is the direct sum of the maps $f_{\mathbf{1}}^{\bar{N}_{R}(S)} \operatorname{Defres}_{\bar{N}_{R}(S)}$. Corollary 4.24 follows from the fact that

$$
f_{\mathbf{1}}^{\bar{N}_{R}(S)} \operatorname{Defres}_{\bar{N}_{R}(S)}^{R}\left(f_{\mathbf{1}}^{P} \times f_{\mathbf{1}}^{Q}\right)=0
$$


unless $S$ is centrally diagonal in $R=P \times Q$ : indeed Defres $\frac{R}{N_{R}(S)}$ is given by the $\left(\bar{N}_{R}(S), R\right)$-biset $S \backslash R$. On the other hand

$$
\begin{aligned}
f_{\mathbf{1}}^{P} \times f_{\mathbf{1}}^{Q} & =\left(P / \mathbf{1}-P / Z_{P}\right) \times\left(Q / \mathbf{1}-Q / Z_{Q}\right) \\
& =R /(\mathbf{1} \times \mathbf{1})-R /\left(\mathbf{1} \times Z_{Q}\right)-R /\left(Z_{P} \times \mathbf{1}\right)+R /\left(Z_{P} \times Z_{Q}\right)
\end{aligned}
$$

hence Defres $\frac{R}{\bar{N}_{R}(S)}\left(f_{\mathbf{1}}^{P} \times f_{\mathbf{1}}^{Q}\right)$ is equal to

$$
S \backslash R /(\mathbf{1} \times \mathbf{1})-S \backslash R /\left(\mathbf{1} \times Z_{Q}\right)-S \backslash R /\left(Z_{P} \times \mathbf{1}\right)+S \backslash R /\left(Z_{P} \times Z_{Q}\right)
$$

which is

$$
S \backslash R-S\left(\mathbf{1} \times Z_{Q}\right) \backslash R-S\left(Z_{P} \times \mathbf{1}\right) \backslash R+S\left(Z_{P} \times Z_{Q}\right) \backslash R
$$

If $S$ is not centrally diagonal in $R=P \times Q$, then either $S=S\left(\mathbf{1} \times Z_{Q}\right)$ or $S=S\left(Z_{P} \times \mathbf{1}\right)$. In each case the sum 4.26 vanishes.

And if $S$ is centrally diagonal in $R$, then

$$
S\left(\mathbf{1} \times Z_{Q}\right)=S\left(Z_{P} \times \mathbf{1}\right)=S\left(Z_{P} \times Z_{Q}\right),
$$

since the image of these groups in the Roquette group $\bar{N}_{R}(S)$ is equal to its unique central subgroup $\widehat{S} / S$ of order $p$. In this case

$$
\text { Defres } \bar{N}_{R}(S)\left(f_{\mathbf{1}}^{P} \times f_{\mathbf{1}}^{Q}\right)=S \backslash R-\widehat{S} \backslash R .
$$

Since $f_{\mathbf{1}}^{\bar{N}_{R}(S)}=N_{R}(S) / S-N_{R}(S) / \widehat{S}$, it follows that $\operatorname{Defres} \frac{R}{\bar{N}_{R}(S)}\left(f_{\mathbf{1}}^{P} \times f_{\mathbf{1}}^{Q}\right)$ is invariant by composition with $f_{1}^{\bar{N}_{R}(S)}$.

Conversely, if $S$ is not centrally diagonal in $(P \times Q)$, then

$$
\left(f_{\mathbf{1}}^{P} \times f_{\mathbf{1}}^{Q}\right) \operatorname{Indinf} \frac{R}{\bar{N}_{R}(S)} f_{\mathbf{1}}^{\bar{N}_{R}(S)}=0
$$

as can be seen by taking opposite bisets in equation 4.25. And if $S$ is centrally diagonal, then

$$
\left(f_{\mathbf{1}}^{P} \times f_{\mathbf{1}}^{Q}\right) \operatorname{Indinf} \frac{R}{\bar{N}_{R}(S)} f_{\mathbf{1}}^{\bar{N}_{R}(S)}=R / S-R / \widehat{S}=\operatorname{Indinf} \frac{R}{\bar{N}_{R}(S)} f_{\mathbf{1}}^{\bar{N}_{R}(S)} .
$$

Hence the isomorphisms $\mathcal{D}$ and $\mathcal{I}$ restrict to mutual inverse isomorphisms between $\partial P \times \partial Q$ and the direct sum of the edges $\partial \bar{N}_{R}(S)$, where $S$ is a centrally diagonal genetic subgroup of $R$. But for all such subgroups $S$, the group $\bar{N}_{R}(S)$ is isomorphic to $P \diamond Q$, and there are $\nu_{P, Q}$ centrally diagonal subgroups in a genetic basis of $R=P \times Q$. This completes the proof. 


\section{Examples and applications}

5.1. Suppose first that $p$ is odd. Then the Roquette $p$-groups are just the cyclic groups $C_{p^{n}}$, for $n \geq 0$. The "multiplication rule" of the edges $\partial C_{p^{n}}$ is the following

$$
\forall m, \forall n \in \mathbb{N}, \partial C_{p^{m}} \times \partial C_{p^{n}}=\phi\left(p^{\min (m, n)}\right) \partial C_{p^{\max (m, n)}},
$$

where $\phi$ is the Euler function (thus $\phi\left(p^{k}\right)=p^{k-1}(p-1)$ if $k>0$, and $\phi(1)=1)$.

5.3. Some surprising phenomenons occur when $p=2$ :

5.4. Proposition : In $\mathcal{R}_{2}$, the edge $\partial C_{2}$ is isomorphic to the trivial group $\mathbf{1}$ (or its edge $\mathbf{0 1}$ ).

Proof : Indeed Corollary 3.12 implies that if $E \cong\left(C_{2}\right)^{2}$, then $\partial E=0$ in $\mathcal{R}_{2}$. Let $X, Y$ and $Z$ denote the subgroups of order 2 of $E$. The element

$$
u=\operatorname{Res}_{X}^{E} \times_{E} f_{1}^{E} \times_{E} \operatorname{Ind}_{Y}^{E}
$$

of $B(X, Y)$ can be viewed as a morphism from $Y$ to $X$ in the category $\mathcal{R}_{2}$, which factors through $\partial E$. So this morphism is equal to 0 . Since

$$
f_{\mathbf{1}}^{E}=E / \mathbf{1}-E / X-E / Y-E / Z+2 E / E,
$$

it follows that

$$
u=\operatorname{Ind}_{1}^{X} \operatorname{Res}_{1}^{Y}-\operatorname{Inf}_{1}^{X} \operatorname{Res}_{1}^{Y}-\operatorname{Ind}_{1}^{X} \operatorname{Def}_{1}^{Y}-\operatorname{Iso}(\varphi)+2 \operatorname{Inf}_{1}^{X} \operatorname{Def}_{1}^{Y},
$$

where $\varphi$ is the unique group isomorphism from $Y$ to $X$. Thus

$0=u \operatorname{Iso}\left(\varphi^{-1}\right)=\operatorname{Ind}_{1}^{X} \operatorname{Res}_{1}^{X}-\operatorname{Inf}_{1}^{X} \operatorname{Res}_{1}^{X}-\operatorname{Ind}_{1}^{X} \operatorname{Def}_{1}^{X}-\operatorname{Id}_{X}+2 \operatorname{Inf}_{1}^{X} \operatorname{Def}_{1}^{X}$.

Hence in the category $\mathcal{R}_{2}$

$$
\operatorname{Id}_{X}=\left(\operatorname{Ind}_{1}^{X}-\operatorname{Inf}_{1}^{X}\right)\left(\operatorname{Res}_{1}^{X}-\operatorname{Def}_{1}^{X}\right)+\operatorname{Inf}_{1}^{X} \operatorname{Def}_{1}^{X} .
$$

It follows that

$$
f_{1}^{X}=f_{1}^{X}\left(\operatorname{Ind}_{1}^{X}-\operatorname{Inf}_{1}^{X}\right)\left(\operatorname{Res}_{1}^{X}-\operatorname{Def}_{1}^{X}\right) f_{1}^{X} .
$$

But on the other hand $f_{1}^{X}=\operatorname{Id}_{X}-\operatorname{Inf}_{1}^{X} \operatorname{Def}_{1}^{X}$, so

$$
\begin{aligned}
f_{1}^{X}\left(\operatorname{Ind}_{1}^{X}-\operatorname{Inf}_{1}^{X}\right) & =f_{1}^{X} \operatorname{Ind}_{1}^{X} \\
& =\operatorname{Ind}_{1}^{X}-\operatorname{Inf}_{1}^{X} \operatorname{Def}_{1}^{X} \operatorname{Ind}_{1}^{X} \\
& =\operatorname{Ind}_{1}^{X}-\operatorname{Inf}_{1}^{X} .
\end{aligned}
$$


It follows that

$$
\begin{aligned}
\left(\operatorname{Res}_{1}^{X}-\operatorname{Def}_{1}^{X}\right) f_{1}^{X}\left(\operatorname{Ind}_{1}^{X}-\operatorname{Inf}_{1}^{X}\right) & =\left(\operatorname{Res}_{1}^{X}-\operatorname{Def}_{1}^{X}\right)\left(\operatorname{Ind}_{1}^{X}-\operatorname{Inf}_{1}^{X}\right) \\
& =2 \mathrm{Id}_{1}-\mathrm{Id}_{1}-\mathrm{Id}_{1}+\mathrm{Id}_{1} \\
& =\mathrm{Id}_{\mathbf{1}} .
\end{aligned}
$$

Thus, setting

$$
\begin{aligned}
a & =f_{1}^{X}\left(\operatorname{Ind}_{1}^{X}-\operatorname{Inf}_{1}^{X}\right) \in \operatorname{Hom}_{\mathcal{R}_{2}}(\mathbf{1}, \partial X) \\
b & =\left(\operatorname{Res}_{1}^{X}-\operatorname{Def}_{\mathbf{1}}^{X}\right) f_{\mathbf{1}}^{X} \in \operatorname{Hom}_{\mathcal{R}_{2}}(\partial X, \mathbf{1})
\end{aligned}
$$

the composition $b \circ a$ is equal to $\mathrm{Id}_{1}$, and Equation 5.5 shows that the composition $a \circ b$ is equal to the identity of $\partial X$. So $a$ and $b$ are mutual inverse isomorphisms between 1 and $\partial X$.

5.6. Corollary : Let $F$ be a rational 2-biset functor. Then for any finite 2-group $P$

$$
\begin{gathered}
F\left(C_{2} \times P\right) \cong F(P) \oplus F(P) . \\
F\left(D_{8} \times P\right) \cong F(P)^{\oplus 5} .
\end{gathered}
$$

Proof : Indeed rational $p$-biset functors are exactly those $p$-biset functors which factor through the category $\mathcal{R}_{p}$. And in the category $\mathcal{R}_{2}$, by Theorem 3.11, there is an isomorphism

$$
C_{2} \cong \mathbf{1} \oplus \partial C_{2} \cong \mathbf{1} \oplus \mathbf{1}
$$

Thus $C_{2} \times P \cong P \oplus P$, and the first assertion follows. The second one follows from Example 3.13, which shows that in $\mathcal{R}_{2}$

$$
D_{8} \cong \mathbf{1} \oplus 4 \cdot \partial C_{2} \cong 5 \cdot \mathbf{1} \text {. }
$$

Hence $D_{8} \times P \cong 5 \cdot P$, thus $F\left(D_{8} \times P\right) \cong F(P)^{\oplus 5}$.

5.7. Proposition : The edge $\partial Q_{8}$ is an involution : more precisely

$$
\partial Q_{8} \times \partial Q_{8}=\partial C_{2} \cong \mathbf{1}
$$

Proof : Indeed $Q_{8} \diamond Q_{8}=C_{2}$, and $\nu_{Q_{8}, Q_{8}}=1$, by Theorem 4.21, 
5.8. Remark : The "action" of this involution on the edges of the other Roquette 2-groups (that is, different from $\mathbf{1}, C_{2}$, and $Q_{8}$ ) is as follows : it stabilizes cyclic and semidihedral groups, and exchanges dihedral and generalized quaternion groups. More precisely, it follows from Corollary 4.24 that

$$
\begin{aligned}
\forall n \geq 2, \partial Q_{8} \times \partial C_{2^{n}} & =\partial C_{2^{n}} \\
\forall n \geq 4, \partial Q_{8} \times \partial D_{2^{n}} & =\partial Q_{2^{n}} \\
\forall n \geq 4, \partial Q_{8} \times \partial Q_{2^{n}} & =\partial D_{2^{n}} \\
\forall n \geq 4, \partial Q_{8} \times \partial S D_{2^{n}} & =\partial S D_{2^{n}}
\end{aligned}
$$

5.9. By Theorem [3.11, any finite $p$-group is isomorphic to a direct sum of edges of Roquette $p$-groups in the category $\mathcal{R}_{p}$. The following result shows that the summands of such an arbitrary direct sum are unique, up to group isomorphism, with the possible exception of the isomorphism $\mathbf{1}=\partial \mathbf{1} \cong \partial C_{2}$ of Proposition 5.4:

5.10. Proposition : Let $\mathcal{S}$ and $\mathcal{T}$ be finite sequences of Roquette p-groups, such that there exists an isomorphism

$$
\underset{S \in \mathcal{S}}{\oplus} \partial S \cong \underset{T \in \mathcal{T}}{\oplus} \partial T
$$

in the category $\mathcal{R}_{p}$. If $p=2$, replace any occurrence of $C_{2}$ in $\mathcal{S}$ and $\mathcal{T}$ by the trivial group, which does not change the existence of the isomorphism 5.11, by Proposition 5.4.

Then there exists a bijection $\varphi: \mathcal{S} \rightarrow \mathcal{T}$ such that the groups $S$ and $\varphi(S)$ are isomorphic, for any $S \in \mathcal{S}$.

Proof : By [3], the simple biset functors $S_{R, \mathbb{F}_{p}}$, where $R$ is a Roquette $p$-group different from $C_{p}$, are rational biset functors. Moreover, if $|R| \geq p^{2}$, then for any finite $p$-group $P$, the dimension of $S_{R, \mathbb{F}_{p}}(P)$ is equal to the number of groups $S$ in a genetic basis of $P$ such that $\bar{N}_{P}(S) \cong R$. On the other hand, the $\mathbb{F}_{p}$-dimension of $S_{1, \mathbb{F}_{p}}(P)$ is equal to the number of groups $S$ in a genetic basis of $P$ such that $\left|\bar{N}_{P}(S)\right| \leq p$.

The functor $S_{R, \mathbb{F}_{p}}$ extends to an additive functor from $\mathcal{R}_{p}$ to the category of $\mathbb{F}_{p}$-vector spaces, and the value of this functor at the edge $\partial P$ is by definition equal to $\partial S_{R, \mathbb{F}_{p}}(P)$. If $|R| \geq p^{2}$, then the $\mathbb{F}_{p^{-}}$dimension of $\partial S_{R, \mathbb{F}_{p}}(P)$ is equal to the number of groups $S$ in a genetic basis of $P$ such that $\bar{N}_{P}(S) \cong R$ 
and $S \cap Z(P)=1$. In particular, if $P$ itself is a Roquette group, then

$$
\operatorname{dim}_{\mathbb{F}_{p}} S_{R, \mathbb{F}_{p}}(\partial P)=\operatorname{dim}_{\mathbb{F}_{p}} \partial S_{R, \mathbb{F}_{p}}(P)= \begin{cases}1 & \text { if } P \cong R \\ 0 & \text { otherwise }\end{cases}
$$

Applying the functor $S_{R, \mathbb{F}_{p}}$ to the isomorphism [5.11, this implies that the number of terms in the sequence $\mathcal{S}$ which are isomorphic to $R$ is equal to the corresponding number in the sequence $\mathcal{T}$.

Similarly, for any finite $p$-group $P$, the $\mathbb{F}_{p}$-dimension of $\partial S_{\mathbf{1}, \mathbb{F}_{p}}(P)$ is equal to the number of groups $S$ in a genetic basis of $P$ such that $\bar{N}_{P}(S) \leq p$ and $S \cap Z(P)=\mathbf{1}$. If $P$ itself is a Roquette group, this gives

$$
\operatorname{dim}_{\mathbb{F}_{p}} S_{\mathbf{1}, \mathbb{F}_{p}}(\partial P)=\operatorname{dim}_{\mathbb{F}_{p}} \partial S_{\mathbf{1}, \mathbb{F}_{p}}(P)= \begin{cases}1 & \text { if } P \cong C_{p} \\ 1 & \text { if } P \cong \mathbf{1} \\ 0 & \text { otherwise }\end{cases}
$$

Hence the number of terms in the sequence $\mathcal{S}$ which are isomorphic to 1 or $C_{p}$ is equal to the corresponding number in the sequence $\mathcal{T}$. If $p=2$, there are no $S$ in $\mathcal{S} \cup \mathcal{T}$ such that $S \cong C_{2}$, by assumption. It follows that for any Roquette $p$-group $R$, the number of terms in the sequence $\mathcal{S}$ which are isomorphic to $R$ is equal to the corresponding number in the sequence $\mathcal{T}$. The proposition follows in this case.

If $p>2$, the above argument shows that

$$
\underset{\substack{S \in \mathcal{S} \\|S| \geq p^{2}}}{\oplus} \partial S \cong \underset{\substack{T \in \mathcal{T} \\|T| \geq p^{2}}}{\oplus} \partial T
$$

Let $M$ denote this direct sum. The isomorphism 5.11 can be rewritten as

$$
m_{\mathbf{1}} \mathbf{1} \oplus m_{C_{p}} \partial C_{p} \oplus M \cong n_{\mathbf{1}} \mathbf{1} \oplus n_{C_{p}} \partial C_{p} \oplus M
$$

for some integers $m_{\mathbf{1}}, m_{C_{p}}, n_{\mathbf{1}}, n_{\mathbb{C}_{p}}$ such that $m_{\mathbf{1}}+m_{C_{p}}=n_{\mathbf{1}}+n_{C_{p}}$.

Now, let $\zeta$ be a primitive (i.e. non-trivial, since $p$ is prime) character $(\mathbb{Z} / p \mathbb{Z})^{\times} \rightarrow \mathbb{C}$. Such a character exists since $p>2$. The functor $S_{C_{p}, \zeta}$ is a rational $p$-biset functor, as it is a summand of $\mathbb{C} R_{\mathbb{C}}$ ([8] Corollary 7.3.5). Applying this functor to the isomorphism 5.12 and taking dimensions gives

$$
m_{C_{p}}+\operatorname{dim}_{\mathbb{C}} S_{C_{p}, \zeta}(M)=n_{C_{p}}+\operatorname{dim}_{\mathbb{C}} S_{C_{p}, \zeta}(M)
$$

since $S_{C_{p}, \zeta}(\mathbf{1})=0$ and $S_{C_{p}, \zeta}\left(C_{p}\right)=\partial S_{C_{p}, \zeta}\left(C_{p}\right) \cong \mathbb{C}$.

It follows that $m_{C_{p}}=n_{C_{p}}$, hence $m_{\mathbf{1}}=n_{\mathbf{1}}$, which completes the proof. 
5.13. Corollary : Let $X, Y$, and $Z$ be objects of $\mathcal{R}_{p}$, isomorphic to direct sums of edges of Roquette p-groups.

1. If $X \oplus Z \cong Y \oplus Z$ in $\mathcal{R}_{p}$, then $X \cong Y$.

2. If $n$ is a positive integer, and if $n \cdot X \cong n \cdot Y$ in $\mathcal{R}_{p}$, then $X \cong Y$.

Proof : Decompose $X$ as $X \cong \bigoplus_{R} n_{R}(X) \cdot \partial R$, where $R$ runs through the set of isomorphism classes of Roquette $p$-groups, and the function $R \mapsto n_{R}(X) \in \mathbb{N}$ has finite support. Choose similar decompositions $Y \cong \underset{R}{\bigoplus_{R}} n_{R}(Y) \cdot \partial R$ and $Z \cong \underset{R}{\oplus_{R}} n_{R}(Z) \cdot \partial R$

For Assertion 1, if $p>2$, it follows from Proposition 5.10 that

$$
n_{R}(X)+n_{R}(Z)=n_{R}(Y)+n_{R}(Z),
$$

for each $R$. Thus $n_{R}(X)=n_{R}(Y)$ for each $R$, hence $X \cong Y$ in $\mathcal{R}_{p}$.

If $p=2$, and if $R$ is a Roquette $p$-group different from 1 and $C_{2}$, Proposition 5.10 shows that $n_{R}(X)+n_{R}(Z)=n_{R}(Y)+n_{R}(Z)$, hence $n_{R}(X)=$ $n_{R}(Y)$. Proposition 5.10 also implies that

$$
n_{\mathbf{1}}(X)+n_{C_{2}}(X)+n_{\mathbf{1}}(Z)+n_{C_{2}}(Z)=n_{\mathbf{1}}(Y)+n_{C_{2}}(Y)+n_{\mathbf{1}}(Z)+n_{C_{2}}(Z) \text {, }
$$

whence $n_{\mathbf{1}}(X)+n_{C_{2}}(X)=n_{\mathbf{1}}(Y)+n_{C_{2}}(Y)$, and $X \cong Y$ in $\mathcal{R}_{2}$ again, since $1 \cong C_{2}$.

The proof of Assertion 2 is similar : if $p>2$, Proposition 5.10 shows that $n n_{R}(X)=n n_{R}(Y)$, for any $R$, thus $n_{R}(X)=n_{R}(Y)$, and $X \cong Y$. And if $p=2$, the conclusion $n_{R}(X)=n_{R}(Y)$ is valid for $R$ different from 1 and $C_{2}$. Moreover $n\left(n_{1}(X)+n_{C_{2}}(X)\right)=n\left(n_{1}(Y)+n_{C_{2}}(Y)\right)$, hence $n_{1}(X)+n_{C_{2}}(X)=$ $n_{\mathbf{1}}(Y)+n_{C_{2}}(Y)$, and $X \cong Y$, since $\mathbf{1} \cong C_{2}$.

In the case of the decomposition of a $p$-group as a direct sum of edges of Roquette groups, the above isomorphism $\partial C_{2} \cong \partial \mathbf{1}$ doesn't matter, and the decomposition is unique :

5.14. Proposition : Let $P$ and $Q$ be finite p-groups. The following assertions are equivalent:

1. The groups $P$ and $Q$ are isomorphic in the category $\mathcal{R}_{p}$.

2. There exist genetic bases $\mathcal{B}_{P}$ and $\mathcal{B}_{Q}$ of $P$ and $Q$, respectively, and a bijection $\sigma: \mathcal{B}_{P} \stackrel{\cong}{\longrightarrow} \mathcal{B}_{Q}$ such that

$$
\forall S \in \mathcal{B}_{P}, \quad N_{Q}(\sigma(S)) / \sigma(S) \cong N_{P}(S) / S
$$


3. For any genetic bases $\mathcal{B}_{P}$ and $\mathcal{B}_{Q}$ of $P$ and $Q$, respectively, there exists a bijection $\sigma: \mathcal{B}_{P} \stackrel{\cong}{\longrightarrow} \mathcal{B}_{Q}$ such that 5.15 holds.

Proof : Assertion 2 implies Assertion 1 by Theorem 3.11. Now suppose that Assertion 1 holds. Then in particular $F(P) \cong F(Q)$, for any rational $p$-biset functor $F$. Let $\mathcal{B}_{P}$ and $\mathcal{B}_{Q}$ be genetic bases of $P$ and $Q$, respectively. If $R$ is a Roquette $p$-group, set

$$
m_{P}(R)=\left|\left\{S \in \mathcal{B} \mid N_{P}(S) / S \cong R\right\}\right|,
$$

and define similarly $m_{Q}(R)$ for the group $Q$. The integers $m_{P}(R)$ and $m_{Q}(R)$ do not depend on the choices of the genetic bases $\mathcal{B}_{P}$ and $\mathcal{B}_{Q}$.

If $R$ is not isomorphic to $C_{p}$, then the simple functor $S_{R, \mathbb{F}_{p}}$ is rational. Moreover, the $\mathbb{F}_{p}$-dimension of $S_{R, \mathbb{F}_{p}}(P)$ is equal to $m_{P}(R)$ if $|R|>p$, and to $1+m_{P}\left(C_{p}\right)$, if $R=1$. Since $P$ is the only element $S$ of $\mathcal{B}$ such that $N_{P}(S) / S=\mathbf{1}$, it follows $m_{P}(\mathbf{1})=1$, and then $m_{P}(R)=m_{Q}(R)$ for any Roquette $p$-group $R$. Assertion 2 follows. The equivalence of Assertions 2 and 3 follows from Theorem 2.22 ,

\subsection{Examples :}

- Let $p>2$, and let $X^{+}$(resp. $X^{-}$) denote the extraspecial $p$-group of order $p^{3}$ and exponent $p$ (resp. $p^{2}$ ). Then $X^{+} \cong X^{-}$in $\mathcal{R}_{p}$, for if $P$ is one of these groups, each genetic basis of $P$ consists of $S=P$, for which $N_{P}(S) / S=\mathbf{1}$, of the $p+1$ subgroups $S$ of index $p$ in $P$, for which $N_{P}(S)=P / S \cong C_{p}$, and an additional non-normal genetic subgroup $S$ such that $N_{P}(S) / S \cong C_{p}$. In other words

$$
X^{+} \cong X^{-} \cong \mathbf{1} \oplus(p+2) \cdot \partial C_{p}
$$

in the category $\mathcal{R}_{p}$.

- Similar examples exist for $p=2$ : if $P$ is one of the groups labelled 6 or 7 in the GAP list of groups of order 32 (see [11]), with respective structure $\left(\left(C_{4} \times C_{2}\right) \rtimes C_{2}\right) \rtimes C_{2}$ and $\left(C_{8} \rtimes C_{2}\right) \rtimes C_{2}$, then in any genetic basis of $P$, there is a unique group $S(=P)$ such that $N_{P}(S) / S=\mathbf{1}$, there are 6 groups $S$ such that $N_{P}(S) / S \cong C_{2}$, and 2 groups $S$ such that $N_{P}(S) / S \cong C_{4}$.

- Some 2-groups with different orders may become isomorphic in the category $\mathcal{R}_{2}$ : using GAP, one can show that the elementary abelian group of order 16 is isomorphic to each of the groups labelled 134, 138, and 177 in GAP's list of groups of order 64 . These groups have respective structure

$$
\left(\left(C_{4} \times C_{4}\right) \rtimes C_{2}\right) \rtimes C_{2}, \quad\left(\left(\left(C_{4} \times C_{2}\right) \rtimes C_{2}\right) \rtimes C_{2}\right) \rtimes C_{2}, \quad \text { and }\left(C_{2} \times D_{16}\right) \rtimes C_{2}
$$


I couldn't find any similar example for $p>2$. In this case however, the following result characterizes those $p$-groups which become isomorphic in the category $\mathcal{R}_{p}$ :

5.17. Proposition : Let $p$ be a prime number, and let $P$ and $Q$ be finite p-groups.

1. If $P \cong Q$ in the category $\mathcal{R}_{p}$, then the $\mathbb{Q}$-algebras $Z \mathbb{Q} P$ and $Z \mathbb{Q} Q$ are isomorphic.

2. If $p>2$, and if $Z \mathbb{Q} P$ and $Z \mathbb{Q} Q$ are isomorphic $\mathbb{Q}$-algebras, then $P \cong Q$ in the category $\mathcal{R}_{p}$.

Proof : Let $\mathcal{G}$ be a genetic basis of $P$. For $S \in \mathcal{G}$, let $V(S)$ denote the corresponding simple $\mathbb{Q} P$-module, defined by

$$
V(S)=\operatorname{Indinf} \bar{N}_{P(S)}^{P} \Phi_{\bar{N}_{P}(S)} .
$$

The multiplicity $v_{S}$ of $V(S)$ in the $\mathbb{Q} P$-module $\mathbb{Q} P$ is equal to

$$
v_{S}=\frac{\operatorname{dim}_{\mathbb{Q}} V(S)}{\operatorname{dim}_{\mathbb{Q}} \operatorname{End}_{\mathbb{Q} P}(V(S))} .
$$

As $S$ is a genetic subgroup of $P$, there is an isomorphism of (skew-)fields

$$
\operatorname{End}_{\mathbb{Q} P}(V(S)) \cong \operatorname{End}_{\mathbb{Q} \bar{N}_{P}(S)}\left(\Phi_{\bar{N}_{P}(S)}\right) .
$$

It follows that there is an isomorphism of $\mathbb{Q}$-algebras

$$
\mathbb{Q} P \cong \prod_{S \in \mathcal{G}} M_{v_{S}}\left(\operatorname{End}_{\mathbb{Q} \bar{N}_{P}(S)}\left(\Phi_{\bar{N}_{P}(S)}\right)\right)
$$

Hence

$$
Z \mathbb{Q} P \cong \prod_{S \in \mathcal{G}} Z\left(\operatorname{End}_{\mathbb{Q} \bar{N}_{P}(S)}\left(\Phi_{\bar{N}_{P}(S)}\right)\right)
$$

It shows that the isomorphism type of the $\mathbb{Q}$-algebra $Z \mathbb{Q} P$ depends only on the genetic basis $\mathcal{G}$ : more precisely, it is determined by the isomorphism type of $P$ in $\mathcal{R}_{p}$. This proves Assertion 1 .

Now if $p>2$, the group $\bar{N}_{P}(S)$ is cyclic, of order $p^{m_{S}}$, say. By Example 2.11, there is an isomorphism of (skew-)fields

$$
\operatorname{End}_{\mathbb{Q} \bar{N}_{P}(S)}\left(\Phi_{\bar{N}_{P}(S)}\right) \cong \mathbb{Q}\left(\zeta_{p^{m_{S}}}\right)
$$


where $\zeta_{p^{m_{S}}}$ is a primitive root of unity of order $p^{m_{S}}$. Hence

$$
Z \mathbb{Q} P \cong \prod_{S \in \mathcal{G}} \mathbb{Q}\left(\zeta_{p^{m}}\right)
$$

Similarly, if $\mathcal{H}$ is a genetic basis of $Q$

$$
Z \mathbb{Q} Q \cong \prod_{T \in \mathcal{H}} \mathbb{Q}\left(\zeta_{p^{n_{T}}}\right)
$$

where $p^{n_{T}}=\left|\bar{N}_{Q}(T)\right|$.

Let $l$ be an integer bigger than all the $m_{S}$ 's, for $S \in \mathcal{G}$, and all the $n_{T}$ 's, for $T \in \mathcal{H}$. Set $K=\mathbb{Q}\left(\zeta_{p^{l}}\right)$, and let $G$ be the Galois group of $K$ over $\mathbb{Q}$. By Galois theory ([15] Theorem 1.5.4 and Remark 1.5.5), the $\mathbb{Q}$-algebras $Z \mathbb{Q} P$ and $Z \mathbb{Q} Q$ are isomorphic if and only if there is an isomorphism of $G$-sets

$$
\operatorname{Hom}_{\mathrm{alg}}(Z \mathbb{Q} P, K) \cong \operatorname{Hom}_{\mathrm{alg}}(Z \mathbb{Q} Q, K)
$$

When $r \leq l$ is an integer, let $G_{r}$ denote the Galois group of $K$ over $\mathbb{Q}\left(\zeta_{p^{r}}\right)$. Then the $G$-set $\operatorname{Hom}_{\text {alg }}(Z \mathbb{Q} P, K)$ is isomorphic to

$$
\bigsqcup_{S \in \mathcal{G}} G / G_{n_{S}}
$$

The isomorphism $Z \mathbb{Q} P \cong Z \mathbb{Q} Q$ implies that for any $r \leq l$, the number of $S \in \mathcal{G}$ such that $\bar{N}_{P}(S)$ has order $p^{r}$ is equal to the number of $T \in \mathcal{H}$ such that $\bar{N}_{Q}(T)$ has order $p^{r}$. Now Assertion 2 of the proposition follows from Proposition 5.14.

5.18. Remark : Assertion 2 of Proposition 5.17 is not true for $p=2$ : let $P=D_{8}$ and $Q=Q_{8}$ denote a dihedral group of order 8 and a quaternion group of order 8 , respectively. By Example 3.13, in a genetic basis of $P$, there is one group $S$ such that $N_{P}(S) / S=1$ (namely $S=P$ ), and 4 subgroups $S$ such that $N_{P}(S) / S \cong C_{2}$ (the 3 subgroups of index 2 in $P$, and a non-central subgroup of order 2 of $P$ ). It follows easily that

$$
\mathbb{Q} D_{8} \cong \mathbb{Q} \oplus \mathbb{Q} \oplus \mathbb{Q} \oplus \mathbb{Q} \oplus M_{2}(\mathbb{Q})
$$

On the other hand, a genetic basis of $Q$ contains one subgroup $S$ such that $N_{Q}(S) / S=\mathbf{1}$ (namely $S=Q$ ), 3 subgroups $S$ such that $N_{Q}(S) / S \cong C_{2}$ (the 3 subgroups of index 2 in $Q$ ), and one subgroup $S$ such that $N_{Q}(S) / S \cong Q_{8}$ (the trivial subgroup of $Q$ ). Hence

$$
\mathbb{Q} Q_{8} \cong \mathbb{Q} \oplus \mathbb{Q} \oplus \mathbb{Q} \oplus \mathbb{Q} \oplus \mathbb{H}_{\mathbb{Q}},
$$


where $\mathbb{H}_{\mathbb{Q}}$ is the field of quaternions over $\mathbb{Q}$. Then

$$
Z \mathbb{Q} D_{8} \cong \mathbb{Q}^{5} \cong Z \mathbb{Q} Q_{8}
$$

But $D_{8}$ and $Q_{8}$ are not isomorphic in $\mathcal{R}_{2}$, by Proposition 5.14 .

5.19. Genetic bases of direct products. Theorem 4.21 yields a way to compute a genetic basis of a direct products of $p$-groups. More precisely:

5.20. Theorem : Let $P$ and $Q$ be finite p-groups, let $\mathcal{B}_{P}$ be a genetic basis of $P$, and let $\mathcal{B}_{Q}$ be a genetic basis of $Q$.

1. For each pair $(S, T) \in \mathcal{B}_{P} \times \mathcal{B}_{Q}$, let $\bar{R}$ be a centrally diagonal genetic subgroup of $\bar{N}_{P}(S) \times \bar{N}_{Q}(T)$, and let

$$
R=\left\{(x, y) \in N_{P}(S) \times N_{Q}(T) \mid(x S, y T) \in \bar{R}\right\} .
$$

Then $R$ is a genetic subgroup of $P \times Q$, such that

$$
\bar{N}_{P \times Q}(R) \cong \bar{N}_{P}(S) \diamond \bar{N}_{Q}(T) \text {. }
$$

2. For $(S, T) \in \mathcal{B}_{P} \times \mathcal{B}_{Q}$, let $\mathcal{E}_{S, T}$ denote the set of subgroups $R$ obtained in Assertion 1, when $\bar{R}$ runs through a set of representatives of centrally diagonal genetic subgroups of $\bar{N}_{P}(S) \times \bar{N}_{Q}(T)$, for the relation $\widehat{=}_{\bar{N}_{P}(S) \times \bar{N}_{Q}(T)}$, as described in Theorem 4.21.

Then the sets $\mathcal{E}_{S, T}$ consist of mutually inequivalent genetic subgroups of $P \times Q$, for the relation $\widehat{=}_{P \times Q}$, and the (disjoint) union

$$
\mathcal{B}_{P \times Q}=\bigsqcup_{(S, T) \in \mathcal{B}_{P} \times \mathcal{B}_{Q}} \mathcal{E}_{S, T}
$$

is a genetic basis of $(P \times Q)$.

Proof : Assertion 1 is straightforward if the group $\bar{N}_{P}(S)$ is trivial, i.e. if $S=P$, or if the group $\bar{N}_{Q}(T)$ is trivial, i.e. if $T=Q$. So we can assume that $S<P$ and $T<Q$.

By Theorem 4.6, the group $\bar{R}$ is diagonal in $\bar{N}_{P}(S) \times \bar{N}_{Q}(T)$. It follows that $k_{1}(R)=S, k_{2}(R)=T$, and $\bar{R}=R /(S \times T)$. This implies in particular that $N_{P \times Q}(R) \leq N_{P}(S) \times N_{Q}(T)$. More precisely

$$
N_{P \times Q}(R)=\left\{(a, b) \in N_{P}(S) \times N_{Q}(T) \mid(a S, b T) \in N_{\bar{N}_{P}(S) \times \bar{N}_{Q}(T)}(\bar{R})\right\},
$$


and the map $(a, b) \mapsto(a S, b T)$ induces a group isomorphism

$$
N_{P \times Q}(R) / R \cong N_{\bar{N}_{P}(S) \times \bar{N}_{Q}(T)}(\bar{R}) / \bar{R} .
$$

It follows that $N_{P \times Q}(R) / R$ is a Roquette group, and by Theorem 4.6 again, and Notation 4.18

$$
N_{P \times Q}(R) / R \cong \bar{N}_{P}(S) \diamond \bar{N}_{Q}(T)
$$

Let $\widehat{S} \geq S$ denote the subgroup of $N_{P}(S)$ such that $\widehat{S} / S$ is the unique central subgroup of order $p$ of the Roquette group $\bar{N}_{P}(S)$. Define $\widehat{T} \geq T$ similarly, and let $\widehat{R} / R$ be the unique central subgroup of order $p$ of $N_{P \times Q}(R) / R$. Then

$$
\widehat{R}=(\widehat{S} \times \mathbf{1}) R=(\mathbf{1} \times \widehat{T}) R .
$$

Let $(x, y) \in P \times Q$ such that $R^{(x, y)} \cap \widehat{R} \leq R$. Intersecting this inclusion with $P \times 1$ gives

$$
\left(S^{x} \cap \widehat{S}\right) \times \mathbf{1} \leq S \times \mathbf{1},
$$

thus $S^{x} \cap \widehat{S} \leq S$, and it follows that $x \in N_{P}(S)$, since $S$ is an expansive subgroup of $P$. Similarly, intersecting the inclusion $R^{(x, y)} \cap \widehat{R} \leq R$ with $1 \times Q$ gives $T^{y} \cap \widehat{T} \leq T$, hence $y \in N_{Q}(T)$.

Now $S \times T \leq R^{(x, y)} \cap \widehat{R} \leq R$, and taking the quotient by $S \times T$ gives

$$
\bar{R}^{(x S, y T)} \cap(\widehat{R} /(S \times T)) \leq \bar{R} .
$$

As $\bar{R}$ is a genetic subgroup of $\bar{N}_{P}(S) \times \bar{N}_{Q}(T)$, it follows that $\bar{R}^{(x S, y T)}$ is equal to $\bar{R}$, hence $R^{(x, y)}=R$. Thus $R$ is an expansive subgroup of $P \times Q$. Since $N_{P \times Q}(R) / R$ is a Roquette group, the group $R$ is a genetic subgroup of $P \times Q$, and this completes the proof of Assertion 1 .

For Assertion 2, let $(S, T)$ and $\left(S^{\prime}, T^{\prime}\right)$ in $\mathcal{B}_{P} \times \mathcal{B}_{Q}$, and let $R \in \mathcal{E}_{S, T}$ and $R^{\prime} \in \mathcal{E}_{S^{\prime}, T^{\prime}}$, such that $R \widehat{=}_{P \times Q} R^{\prime}$. It means that there exists $(x, y) \in P \times Q$ such that

$$
R^{(x, y)} \cap \widehat{R}^{\prime} \leq R^{\prime} \quad, \quad{ }^{(x, y)} R^{\prime} \cap \widehat{R} \leq R
$$

Intersecting these two inclusions with $P \times \mathbf{1}$ gives

$$
S^{x} \cap \widehat{S}^{\prime} \leq S^{\prime},{ }^{x} S^{\prime} \cap \widehat{S} \leq S .
$$

Hence $S^{\prime} \widehat{ }_{P} S$, thus $S^{\prime}=S$, since $S$ and $S^{\prime}$ are in the same genetic basis of $P$. Moreover $x \in N_{P}(S)$. Similarly, intersecting [5.21 with $1 \times Q$ implies $T=T^{\prime}$, and $y \in N_{Q}(T)$. 
Quotienting the inclusions 5.21 by $(S \times T)$ gives that $\bar{R}^{\prime} \widehat{=}_{\bar{N}_{P}(S) \times \bar{N}_{Q}(T)} \bar{R}$. Hence $R^{\prime}=R$, as was to be shown.

Now setting

$$
\mathcal{B}_{P \times Q}=\bigsqcup_{(S, T) \in \mathcal{B}_{P} \times \mathcal{B}_{Q}} \mathcal{E}_{S, T}
$$

yields a set of genetic subgroups of $P \times Q$, which are inequivalent to one other for the relation $\widehat{C}_{P \times Q}$. But

$$
\left|\mathcal{E}_{S, T}\right|=\nu_{\bar{N}_{P}(S), \bar{N}_{Q}(T)},
$$

and $N_{P \times Q}(R) / R \cong \bar{N}_{P}(S) \diamond \bar{N}_{Q}(T)$, for any $R \in \mathcal{E}_{S, T}$ it follows that

$$
\begin{aligned}
\underset{R \in \mathcal{B}_{P \times Q}}{\bigoplus} \partial N_{P \times Q}(R) / R & \cong \underset{\substack{S \in \mathcal{B}_{P} \\
T \in \mathcal{B}_{Q}}}{\bigoplus} \nu_{\bar{N}_{P}(S), \bar{N}_{Q}(T)} \partial\left(\bar{N}_{P}(S) \diamond \bar{N}_{Q}(T)\right) \\
& \cong\left(\underset{S \in \mathcal{B}_{P}}{\bigoplus_{P}} \partial \bar{N}_{P}(S)\right) \times\left(\underset{T \in \mathcal{B}_{Q}}{\oplus} \partial \bar{N}_{Q}(T)\right) \\
& \cong P \times Q .
\end{aligned}
$$

In particular, the rank $l_{\mathbb{Q}}(P \times Q)$ of the group $R_{\mathbb{Q}}(P \times Q)$ is equal to $\left|\mathcal{B}_{P \times Q}\right|$. Since $\mathcal{B}_{P \times Q}$ is contained in a genetic basis of $P \times Q$, which has cardinality $l_{\mathbb{Q}}(P \times Q)$, it follows that $\mathcal{B}_{P \times Q}$ is a genetic basis of $P \times Q$.

5.22. Remark : Theorem 5.20 does not mean that any genetic subgroup of $P \times Q$ can be obtained by the construction of Assertion 1. For example, if $[P, P] \leq Z(P)$ and $Z(P)$ is cyclic, then the diagonal $R=\Delta(P)$ is a genetic subgroup of $P \times P$, by Example2.17. But $k_{1}(R)=1$ is not a genetic subgroup of $P$, if $P$ is not a Roquette group.

5.23. Example of application. As explained in Example 3.13, the dihedral group $D_{8}$ splits as

$$
D_{8} \cong \mathbf{1} \oplus 4 \partial C_{2}
$$

in the category $\mathcal{R}_{2}$. By Proposition 5.4, it follows that $D_{8} \cong 5 \cdot 1$ in $\mathcal{R}_{2}$. Hence $\left(D_{8}\right)^{n} \cong 5^{n} \cdot \mathbf{1}$, for any $n \in \mathbb{N}$. In particular, if $F$ is a rational 2-biset functor such that $F(\mathbf{1})=\{0\}$, then $F\left(\left(D_{8}\right)^{n}\right)=\{0\}$. Hence $F(P)=\{0\}$ for any quotient of a direct product of copies of $D_{8}$, by Remark 3.10.

Actually, one can be more precise : since $\partial C_{2} \times \partial C_{2} \cong \partial C_{2}$ by Corollary 4.24, it follows that for any $n \in \mathbb{N}$

$$
\left(D_{8}\right)^{n} \cong \bigoplus_{i=0}^{n}\left(\begin{array}{c}
n \\
i
\end{array}\right) 4^{i} \cdot\left(\partial C_{2}\right)^{i}=\mathbf{1} \oplus \bigoplus_{i=1}^{n}\left(\begin{array}{c}
n \\
i
\end{array}\right) 4^{i} \cdot \partial C_{2} \cong \mathbf{1} \oplus\left(5^{n}-1\right) \cdot \partial C_{2}
$$


It means that a genetic basis of the group $P=\left(D_{8}\right)^{n}$ is made of the group $S=P$, for which $N_{P}(S) / S=\mathbf{1}$, and of $\left(5^{n}-1\right)$ subgroups $S$ for which $N_{P}(S) / S \cong C_{2}$.

In particular, by Theorem 9.5 of [5] (or Corollary 12.10 .3 of [8]), the Dade group of $P$ is torsion free, and so is the Dade group of any factor group of $P$, by Remark 3.10 again. It shows that the Dade group of a central product of any number of copies of $D_{8}$ is torsion free (see Theorem 5.36 for a generalization of this result) : this was proved by Nadia Mazza and me (Theorem 9.2 of [9]). However, the above argument cannot be considered as a new proof of this result, since Theorem 9.5 of [5] relies on Theorem 9.2 of $[9$.

5.26. Edges of central products. Let $P$ and $Q$ be non-trivial finite p-groups. Recall that a central product $P *_{\varphi} Q$ of $P$ and $Q$ is by definition a group of the form $(P \times Q) / \vec{\Delta}_{\varphi}\left(Z_{P}\right)$, where $Z_{P}$ is a central subgroup of order $p$ of $P$, and $\varphi: Z_{P} \hookrightarrow Z(Q)$ is some isomorphism from $Z_{P}$ to some central subgroup $Z_{Q}$ of $Q$.

In the case where $p=2$ and the groups $P$ and $Q$ both have cyclic center, the group $Z_{P}$ is unique, as well as the morphism $\varphi$, so the central product is simply denoted by $P * Q$ in this case.

5.27. Proposition : Let $p$ be a prime number, and let $P$ and $Q$ be nontrivial finite p-groups. Let $Z_{P}\left(\right.$ resp. $\left.Z_{Q}\right)$ denote a central subgroup of order $p$ of $P(\operatorname{resp} . Q)$.

1. If one of the groups $Z(P)$ or $Z(Q)$ is non-cyclic, or if $|Z(P)|>p$ and $|Z(Q)|>p$, then $\partial\left(P *_{\varphi} Q\right)=0$ in $\mathcal{R}_{p}$, for any group isomorphism $\varphi: Z_{P} \rightarrow Z_{Q}$.

2. If $Z(P)$ and $Z(Q)$ are cyclic, and if moreover $Z(P)$ or $Z(Q)$ has or$\operatorname{der} p$, then, in $\mathcal{R}_{p}$,

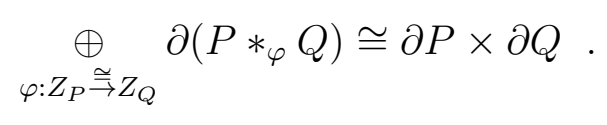

Proof : The center of the group $P *_{\varphi} Q$ is equal to $Z(P) *_{\varphi} Z(Q)$. It is cyclic if and only if both $Z(P)$ and $Z(Q)$ are cyclic, and if one of them has order $p$. This proves Assertion 1.

For Assertion 2, suppose that $Z(P)$ and $Z(Q)$ are cyclic, and that one of them has order $p$. Then the subgroups $Z_{P}$ and $Z_{Q}$ are uniquely determined, and there are $p-1$ group isomorphisms $\varphi: Z_{P} \rightarrow Z_{Q}$. For each of them, the only central subgroup $Z_{\varphi}$ of order $p$ of $P *_{\varphi} Q$ is equal to $\left(Z_{P} \times Z_{Q}\right) / \vec{\Delta}_{\varphi}\left(Z_{P}\right)$, 
and

$$
\left(P *_{\varphi} Q\right) / Z_{\varphi} \cong(P \times Q) /\left(Z_{P} \times Z_{Q}\right) \cong \bar{P} \times \bar{Q}
$$

where $\bar{P}=P / Z_{P}$ and $\bar{Q}=Q / Z_{Q}$.

By Proposition 3.8

$$
\begin{aligned}
& P \times Q \cong \quad \bigoplus_{\mathbf{1} \leq N \unlhd(P \times Q)} \partial((P \times Q) / N) \\
& P *_{\varphi} Q \cong \bigoplus_{\Delta_{\varphi}\left(Z_{P}\right) \leq N \unlhd(P \times Q)} \partial((P \times Q) / N) \\
& \bar{P} \times Q \cong \bigoplus_{\left(Z_{P} \times \mathbf{1}\right) \leq N \unlhd(P \times Q)}^{\oplus} \partial((P \times Q) / N) \\
& P \times \bar{Q} \cong \underset{\left(\mathbf{1} \times Z_{Q}\right) \leq N \unlhd(P \times Q)}{\oplus} \partial((P \times Q) / N) \\
& \bar{P} \times \bar{Q} \cong \underset{\left(Z_{P} \times Z_{Q}\right) \leq N \unlhd(P \times Q)}{\oplus} \partial((P \times Q) / N) .
\end{aligned}
$$

Set

$(5.28)$

$$
S=\partial(P \times Q) \oplus\left(\underset{\varphi: Z_{P} \cong Z_{Q}}{\oplus}\left(P *_{\varphi} Q\right)\right) \oplus(\bar{P} \times Q) \oplus(P \times \bar{Q}) .
$$

Then $S$ is equal to the direct sum of the edges $\partial((P \times Q) / N)$, for $N \unlhd(P \times Q)$, with multiplicity $(p+1)$ if $N \geq\left(Z_{P} \times Z_{Q}\right)$, and multiplicity 1 otherwise. Hence

$$
S \cong(P \times Q)+p \cdot(\bar{P} \times \bar{Q})
$$

Now $\partial(P \times Q)=0$ by Corollary 3.12, Moreover, by Corollary 3.9, for each $\varphi: Z_{P} \stackrel{\cong}{\rightarrow} Z_{Q}$

$$
P *_{\varphi} Q=\partial\left(P *_{\varphi} Q\right) \oplus(\bar{P} \times \bar{Q}) .
$$

But $P \cong \partial P \oplus \bar{P}$ and $Q \cong \partial Q \oplus \bar{Q}$, by Corollary 3.9 again. Replacing $P, Q$, and $P *_{\varphi} Q$ by these values in Equation 5.28 gives

$S \cong\left(\underset{\varphi: Z_{P} \cong Z_{Q}}{\oplus} \partial\left(P *_{\varphi} Q\right)\right) \oplus(p+1) \cdot(\bar{P} \times \bar{Q}) \oplus(\bar{P} \times(\partial Q)) \oplus((\partial P) \times \bar{Q})$.

But replacing $P$ by $\partial P \oplus \bar{P}$ and $Q$ by $\partial Q \oplus \bar{Q}$ in Equation 5.29 gives

(5.31) $S \cong(\partial P \times \partial Q) \oplus(p+1) \cdot(\bar{P} \times \bar{Q}) \oplus(\bar{P} \times(\partial Q)) \oplus((\partial P) \times \bar{Q})$.

Comparing Equations 5.30 and 5.31 gives

$$
\underset{\varphi: Z_{P} \stackrel{\bigoplus \cong}{\rightrightarrows} Z_{Q}}{\oplus} \partial\left(P *_{\varphi} Q\right) \cong \partial P \times \partial Q
$$


by Corollary 5.13, This completes the proof.

\subsection{Corollary :}

1. Let $P$ and $Q$ be non-trivial finite 2-groups with cyclic center, and assume that $Z(P)$ or $Z(Q)$ has order 2. Then

$$
\partial(P * Q) \cong \partial P \times \partial Q
$$

in the category $\mathcal{R}_{2}$.

2. For each $i \in\{1, \ldots, n\}$, let $P_{i}$ be a finite 2-group with center $Z_{i}$ of order 2. Let $\underset{i=1}{*} P_{i}=P_{1} * P_{2} * \cdots * P_{n}$ denote the central product of the groups $P_{i}$. Then

$$
\underset{i=1}{\stackrel{n}{*}} P_{i} \cong \prod_{i=1}^{n}\left(\partial P_{i}\right) \oplus \prod_{i=1}^{n}\left(P_{i} / Z_{i}\right)
$$

in the category $\mathcal{R}_{2}$.

3. In particular, for any positive integer $n$, and any integer $m \geq 4$, there are isomorphisms

$$
\begin{aligned}
\left(D_{2^{m}}\right)^{* n} & \cong 2^{(n-1)(m-3)} \cdot \partial D_{2^{m}} \oplus\left(D_{2^{m-1}}\right)^{n} \\
\left(S D_{2^{m}}\right)^{* n} & \cong 2^{(n-1)(m-3)} \cdot \partial S D_{2^{m}} \oplus\left(D_{2^{m-1}}\right)^{n} \\
\left(Q_{2^{m}}\right)^{* n} & \cong\left\{\begin{array}{l}
2^{(n-1)(m-3)} \cdot \partial D_{2^{m}} \oplus\left(D_{2^{m-1}}\right)^{n} \text { if } n \text { is even } \\
2^{(n-1)(m-3)} \cdot \partial Q_{2^{m}} \oplus\left(D_{2^{m-1}}\right)^{n} \text { if } n \text { is odd }
\end{array}\right.
\end{aligned}
$$

in the category $\mathcal{R}_{2}$, where $P^{* n}$ denote the central product of $n$ copies of $P$.

Proof : For Assertion 1, the assumptions imply that there is a unique isomorphism $\varphi: Z_{P} \rightarrow Z_{Q}$. Hence there is only one term in the summation of Proposition 5.27 .

Assertion 2 follows from Corollary 3.9, which gives an isomorphism

$$
\underset{i=1}{\underset{*}{*}} P_{i} \cong \partial\left(\underset{i=1}{*} P_{i}\right) \oplus\left(\left(\begin{array}{c}
n \\
\underset{i=1}{*} P_{i}
\end{array}\right) / Y\right)
$$

where $Y$ is the unique central subgroup of order $p$ in $\underset{i=1}{\stackrel{n}{*}} P_{i}$. Now an easy 
induction argument, using Assertion 1 , shows that $\partial\left(\underset{i=1}{\underset{*}{*}} P_{i}\right) \cong \prod_{i=1}^{n}\left(\partial P_{i}\right)$, and that $\left(\underset{i=1}{\stackrel{n}{*}} P_{i}\right) / Y \cong \prod_{i=1}^{n}\left(P_{i} / Z_{i}\right)$.

Finally, when $P$ is one of the groups $D_{2^{m}}, S D_{2^{m}}$, or $S D_{2^{m}}$, then $P / Z \cong$ $D_{2^{m-1}}$. Now Assertion 3 follows from Assertion 2 and from an easy induction argument using Corollary 4.24 .

5.33. Remark : It follows from Assertion 3 that when $n$ is even, the groups $\left(D_{2^{m}}\right)^{* n}$ and $\left(Q_{2^{m}}\right)^{* n}$ are isomorphic in the category $\mathcal{R}_{2}$ : it is actually easy to check that they are isomorphic as groups.

5.34. Example : From Corollary 4.24 and Assertion 1, it follows that :

- $\partial\left(\left(D_{8}\right)^{* n}\right) \cong \partial C_{2}$.

- $\partial\left(\left(Q_{8}\right)^{* n}\right) \cong \begin{cases}\partial C_{2} & \text { if } n \text { is even } \\ \partial Q_{8} & \text { if } n \text { is odd }\end{cases}$

- $\partial\left(\left(S D_{2^{m}}\right)^{* n}\right) \cong 2^{(n-1)(m-3)} \cdot \partial S D_{2^{m}}$, for $m \geq 4$.

More generally, if $P$ is any central product of groups isomorphic to $D_{8}$ or $Q_{8}$, that is, if $P$ is an extraspecial 2 -group, then $\partial P \cong \partial C_{2}$, or $\partial P \cong \partial Q_{8}$. In particular (see Example 2.28), we recover the well known fact that $P$ has a unique faithful rational irreducible representation. But there is more : let $Q$ be a non trivial 2-group. If the center of $Q$ is not cyclic, then the center of any central product $P * Q$ is not cyclic, hence $\partial Q=\partial(P * Q)=0$ in $\mathcal{R}_{2}$. If the center of $Q$ is cyclic, then there is a unique central product $P * Q$. By Theorem 3.11, there is a finite sequence $\mathcal{S}$ of Roquette 2 -groups such that

$$
\partial Q \cong \underset{R \in \mathcal{S}}{\oplus} \partial R
$$

in the category $\mathcal{R}_{2}$. By Assertion 1 of Corollary 5.32, it follows that

$$
\partial(P * Q) \cong \underset{R \in \mathcal{S}}{\oplus}(\partial P \times \partial R)
$$

Now $\partial P \cong \partial C_{2}$ or $\partial P \cong \partial Q_{8}$. In both cases, by Proposition 5.4 and Proposition 5.7. the multiplication by $\partial P$ is a permutation of the edges of the Roquette 2-groups. It follows that there is a sequence $\mathcal{S}^{\prime}$ of Roquette 2 groups, of the same length as $\mathcal{S}$, such that

$$
\partial(P * Q) \cong \underset{R \in \mathcal{S}^{\prime}}{\bigoplus} \partial R .
$$

In particular, for any field $K$ of characteristic 0 , the groups $R_{K}(\partial P)=$ $\partial R_{K}(P)$ and $R_{K}(\partial(P * Q))=\partial R_{K}(P * Q)$ are free of the same rank, equal to the length of $\mathcal{S}$ or $\mathcal{S}^{\prime}$. 
Hence in any case, the groups $Q$ and $P * Q$ have the same number (possibly 0 if the center of $Q$ is not cyclic) of faithful irreducible representations over $K$, up to isomorphism.

Similarly, the last example above means in particular that the group $\left(S D_{2^{m}}\right)^{* n}$ admits $2^{(n-1)(m-3)}$ non isomorphic faithful rational irreducible representations.

5.35. Example : Let $p$ be an odd prime, and let $P=X^{\epsilon}$ (where $\epsilon \in\{ \pm 1\}$ ) be one of the extraspecial groups of order $p^{3}$ considered in Examples 5.16. Then $\partial P \cong \partial C_{p}$. Moreover $Z(P)$ has order $p$, and any automorphism of $Z(P)=Z_{P}$ can be extended to an automorphism of $P$. It follows that $P *_{\varphi} Q$ is independent (up to a group isomorphism) of the choice of an embedding $\varphi: P \hookrightarrow Z(Q)$, for any non-trivial $p$-group $Q$ with cyclic center, so we can denote this group by $P * Q$.

Now if $Q$ is a non-trivial $p$-group, and $\mathcal{B}$ is a genetic basis of $Q$, it follows from Theorem 3.11 that

$$
\partial Q \cong \underset{\substack{S \in \mathcal{B} \\ S \cap Z(Q)=\mathbf{1}}}{\oplus} \partial \bar{N}_{Q}(S)
$$

and the right hand side is a direct sum of non-trivial Roquette $p$-groups. By Equation 5.2, for any non trivial Roquette $p$-group $R$

$$
\partial C_{p} \times \partial R \cong(p-1) \partial R
$$

Hence for any non-trivial $p$-group $Q$

$$
\partial C_{p} \times \partial Q \cong(p-1) \partial Q
$$

It follows that if $Q$ is a non-trivial $p$-group with cyclic center, and if $P \cong X^{\epsilon}$, then

$$
\underset{\varphi: Z_{P} \cong Z_{Q}}{\oplus} \partial\left(P *_{\varphi} Q\right) \cong(p-1) \partial(P * Q) \cong \partial C_{p} \times \partial Q \cong(p-1) \partial Q
$$

hence $\partial\left(X^{\epsilon} * Q\right) \cong \partial Q$, by Corollary 5.13. Note that this is also true (for any central product of $X^{\epsilon}$ with $Q$ ) if the center of $Q$ is non-trivial, since in this case the center of $X^{\epsilon} * Q$ is also non trivial, and then $\partial\left(X^{\epsilon} * Q\right) \cong \partial Q \cong 0$ in $\mathcal{R}_{p}$, by Corollary 3.12 ,

It follows easily by induction that if $P$ is any central product of groups isomorphic to $X^{+}$or $X^{-}$, i.e. if $P$ is an extraspecial $p$-group, then $\partial P \cong \partial C_{p}$. We recover this way the well known fact that for $p>2$ also, extraspecial $p$ groups have a unique faithful rational irreducible representation. The same 
argument shows more generally that $\partial(P * Q) \cong \partial Q$ in $\mathcal{R}_{p}$, for any nontrivial $p$-group $Q$. In particular $Q$ and $P * Q$ have the same number of faithful irreducible representations over a given field $K$ of characteristic 0 .

\subsection{Theorem :}

1. Let $P$ be an arbitrary finite direct product of groups of order 2 and dihedral 2-groups. Then the Dade group of any factor group of $P$ is torsion free.

2. Let $4 \leq m_{1} \leq m_{2} \leq \cdots \leq m_{n}$ be a non decreasing sequence of integers. Set $s=\sum_{i=1}^{n-1}\left(m_{i}-3\right)$. Then the torsion part of the Dade group of $\underset{i=1}{*} S D_{2^{m_{i}}}$ is isomorphic to $(\mathbb{Z} / 2 \mathbb{Z})^{2^{s-1}}$ if $m_{1}<m_{n}$, and to $(\mathbb{Z} / 2 \mathbb{Z})^{2^{s}}$ if $m_{1}=m_{n}$.

3. In particular, for any integers $n \geq 1$ and $m \geq 4$, the torsion part of the Dade group of $\left(S D_{2^{m}}\right)^{* n}$ is isomorphic to $(\mathbb{Z} / 2 \mathbb{Z})^{2^{(n-1)(m-3)}}$.

Proof : First by Example 3.13

$$
D_{8} \cong \mathbf{1} \oplus 4 \cdot \partial C_{2}
$$

in $\mathcal{R}_{2}$. Now by Corollary 3.9 , since the center $Z$ of $D_{16}$ has order 2 , and since $D_{16} / Z \cong D_{8}$

$$
D_{16} \cong \mathbf{1} \oplus 4 \cdot \partial C_{2} \oplus \partial D_{16}
$$

Since for $n \geq 3$, the group $D_{2^{n}}$ has a center $Z$ of order 2 , and since $D_{2^{n}} / Z \cong$ $D_{2^{n-1}}$, it follows by induction that

$$
D_{2^{n}} \cong \mathbf{1} \oplus 4 \cdot \partial C_{2} \oplus \bigoplus_{l=4}^{n} \partial D_{2^{l}} .
$$

Now by Corollary 4.24, the product $\partial D_{2^{l}} \times \partial D_{2^{m}}$, for $l \leq m$, is isomorphic to $2^{l-3} \cdot \partial D_{2^{m}}$. Moreover $\partial C_{2} \times \partial P \cong \partial P$ for any 2 -group $P$, since $\partial C_{2} \cong \mathbf{1}$ by Proposition 5.4, it follows that any product of dihedral 2-groups and groups of order 2 is isomorphic to a direct sum of edges of the trivial group, of the edge of the group of order 2 , and of edges of dihedral 2-groups.

It follows that if $P$ is a direct product of dihedral 2-groups and groups of order 2 , then $P$ is isomorphic in $\mathcal{R}_{2}$ to the direct sum of the trivial group, and some copies of edges of the group of order 2 and the edge of dihedral 2-groups. In other words, if $S$ is a genetic subgroup of $P$, then $N_{P}(S) / S$ is either trivial, or of order 2, or dihedral. Now the Dade group of dihedral 
2-groups is torsion free (by [10] Theorem 10.3 (a)), and the Dade groups of the trivial group and the group of order 2 are trivial. It follows that the Dade group of $P$ is torsion free, as well as the Dade group of any quotient of $P$, as is it a direct summand of the Dade group of $P$. This proves Assertion 1.

For Assertion 2, set $P=\underset{i=1}{*} S D_{2^{m_{i}}}$. By Assertion 2 of Corollary [5.32,

$$
P \cong \prod_{i=1}^{m} \partial S D_{2^{m_{i}}} \oplus \prod_{i=1}^{n} D_{2^{m_{i}-1}}
$$

in the category $\mathcal{R}_{2}$. Now an easy induction on $n$, using Corollary 4.24 , shows that

$$
\prod_{i=1}^{n} \partial S D_{2^{m_{i}}} \cong\left\{\begin{array}{cc}
2^{s-1} \cdot \partial C_{2^{m_{n}-1}} & \text { if } m_{1}<m_{n} \\
2^{s} \cdot \partial S D_{2^{m_{n}}} & \text { if } m_{1}=m_{n}
\end{array}\right.
$$

where $s=\sum_{i=1}^{n-1}\left(m_{i}-3\right)$.

By 5.38, this means that in a genetic basis $\mathcal{B}$ of $P$, there are $2^{s-1}$ or $2^{s}$ subgroups $S$ such that $N_{P}(S) / S$ is semidihedral, depending on $m_{1}<m_{n}$ or $m_{1}=m_{n}$, and for the other $S \in \mathcal{B}$, the group $N_{P}(S) / S$ is trivial, of order 2 , or dihedral.

The Dade group of a dihedral 2-group is torsion free, and the Dade groups of the trivial group and $C_{2}$ are trivial. Moreover, the faithful torsion part $\partial D^{t}\left(C_{2^{m}}\right)$ of the Dade group of $C_{2^{m}}$ is isomorphic to $\mathbb{Z} / 2 \mathbb{Z}$, if $m \geq 2$ (see [8] Theorem 12.10.3). Similarly, the faithful torsion part $\partial D^{t}\left(S D_{2^{m}}\right)$, for $m \geq 4$, is isomorphic to $\mathbb{Z} / 2 \mathbb{Z}$. This completes the proof of Assertion 2. Assertion 3 is a particular case of Assertion 2.

5.39. Remark : Let $P$ be a finite product of groups of order 2 , and dihedral 2-groups, as in Assertion 1, and let $Q$ be a quotient of $P$. If $T$ is a genetic subgroup of $Q$, then $N_{Q}(T) / T$ is either trivial, of order 2 , or dihedral : indeed $T$ lifts to a genetic subgroup $S$ of $P$, such that $N_{P}(S) / S \cong N_{Q}(T) / T$. It follows in particular that the map

$$
\bar{\epsilon}_{Q}: B^{\times}(Q) \rightarrow \operatorname{Hom}_{\mathbb{Z}}\left(R_{\mathbb{Q}}(Q), \mathbb{F}_{2}\right)
$$

introduced in [6], Notation 8.4, is a group isomorphism from the group of units of the Burnside ring of $Q$ to the $\mathbb{F}_{2}$-dual of $R_{\mathbb{Q}}(Q)$ : indeed, there are non-negative integers $a$ and $b_{i}$, for $i \in\{4, \ldots, m\}$ such that

$$
Q \cong \mathbf{1} \oplus a \cdot \partial C_{2} \oplus \underset{i=4}{\oplus} b_{i} \cdot \partial D_{2^{i}}
$$


in the category $\mathcal{R}_{2}$. Then $B^{\times}(Q) \cong\left(\mathbb{F}_{2}\right)^{r}$, where $r=1+a+\sum_{i=4}^{m} b_{i}$, by [6], Theorem 8.5. Similarly $R_{\mathbb{Q}}(Q) \cong \mathbb{Z}^{r}$ (hence $r$ is equal to the number of conjugacy classes of cyclic subgroups of $Q)$, so $\operatorname{Hom}_{\mathbb{Z}}\left(R_{\mathbb{Q}}(Q), \mathbb{F}_{2}\right) \cong\left(\mathbb{F}_{2}\right)^{r}$. As $\bar{\epsilon}$ is injective, it is an isomorphism.

5.40. Proposition : Let $m \geq 3$ be an integer. Then for any integer $n$, there is an isomorphism

$$
\left(D_{2^{m}}\right)^{n} \cong \mathbf{1} \oplus\left(5^{n}-1\right) \cdot \partial C_{2} \oplus \bigoplus_{l=4}^{m} \frac{\left(3+2^{l-2}\right)^{n}-\left(3+2^{l-3}\right)^{n}}{2^{l-3}} \cdot \partial D_{2^{l}}
$$

in the category $\mathcal{R}_{2}$.

Proof : Let $\mathcal{S}_{p}$ denote the full subcategory of $\mathcal{R}_{p}$ consisting of finite direct sums of edges of Roquette $p$-groups, and let $\Gamma=K_{0}\left(\mathcal{S}_{p}\right)$ be the Grothendieck group of this category, for relations given by direct sum decomposition. Then Corollary 5.13 shows that $\Gamma$ is a free abelian group, and that two objects of $\mathcal{S}_{p}$ have the same image in $\Gamma$ if and only if they are isomorphic in $\mathcal{R}_{p}$. Moreover, by Corollary 4.24 , the category $\mathcal{S}_{p}$ is a tensor subcategory of $\mathcal{R}_{p}$, and $\Gamma$ is actually a commutative ring.

It follows that $\Gamma$ identifies to a subring of the $\mathbb{Q}$-algebra $\mathbb{Q} \Gamma=\mathbb{Q} \otimes_{\mathbb{Z}} \Gamma$, and that, to prove the proposition, it suffices to check that the two sides of Equation 5.41 have the same image in $\mathbb{Q} \Gamma$. Let $c$ denote the image of $\partial C_{2}$ in $\Gamma$, and for $l \geq 4$, let $d_{l}$ denote the image of $\partial D_{2^{l}}$ in $\Gamma$. By Equation 5.37, the image $i_{m}$ of $D_{2^{m}}$ in $\Gamma$ is equal to

$$
i_{m}=1+4 c+\sum_{l=4}^{m} d_{l}
$$

By Corollary 4.24, for $4 \leq l \leq k$

$$
d_{l} \times d_{k}=2^{l-3} d_{k} .
$$

It follows that the elements $e_{l}=\frac{1}{2^{l-3}} d_{l}$ of $\mathbb{Q} \Gamma$, for $l \geq 4$, are such that

$$
\forall l, k, 4 \leq l \leq k, \quad e_{l} \times e_{k}=e_{k} .
$$

In particular $e_{l}$ is an idempotent, and the elements

$$
f_{l}=e_{l}-e_{l+1}, \text { for } 4 \leq l<m \text {, and } f_{m}=e_{m}
$$


are orthogonal idempotents of $\mathbb{Q} \Gamma$. With this notation, for $l \geq 4$

$$
e_{l}=f_{l}+f_{l+1}+\cdots+f_{m},
$$

and the element $i_{r}$ can be written as

$$
\begin{aligned}
i_{m} & =1+4 c+\sum_{l=4}^{m} d_{l} \\
& =1+4 c+\sum_{l=4}^{m} 2^{l-3}\left(f_{l}+f_{l+1}+\cdots+f_{m}\right) \\
& =1+4 c+\sum_{l=4}^{m} 2\left(2^{l-3}-1\right) f_{l} .
\end{aligned}
$$

Moreover, it follows from Proposition 5.4 that $c \times f_{l}=f_{l}$, for $4 \leq l \leq m$. Thus

$$
\begin{aligned}
\left(i_{m}\right)^{n}= & (1+4 c)^{n}+\sum_{j=1}^{n}\left(\begin{array}{c}
n \\
j
\end{array}\right)(1+4 c)^{n-j}\left(\sum_{l=4}^{m} 2\left(2^{l-3}-1\right) f_{l}\right)^{j} \\
= & (1+4 c)^{n}+\sum_{j=1}^{n}\left(\begin{array}{c}
n \\
j
\end{array}\right) 5^{n-j} \sum_{l=4}^{m} 2^{j}\left(2^{l-3}-1\right)^{j} f_{l} \\
= & (1+4 c)^{n}+\sum_{l=4}^{m}\left(\sum_{j=1}^{n}\left(\begin{array}{c}
n \\
j
\end{array}\right) 5^{n-j} 2^{j}\left(2^{l-3}-1\right)^{j}\right) f_{l} \\
\left(i_{m}\right)^{n} & =(1+4 c)^{n}+\sum_{l=4}^{m}\left(\left(5+2\left(2^{l-3}-1\right)\right)^{n}-5^{n}\right) f_{l} \\
& =(1+4 c)^{n}+\sum_{l=4}^{m}\left(\left(3+2^{l-2}\right)^{n}-5^{n}\right) f_{l} \\
& =(1+4 c)^{n}+\sum_{l=4}^{m}\left(\left(3+2^{l-2}\right)^{n}-\left(3+2^{l-3}\right)^{n}\right) e_{l}
\end{aligned}
$$

The proposition follows, since $(1+4 c)^{n}=1+\left(5^{n}-1\right) c$, by Equation 5.25, and since $e_{l}=\frac{1}{2^{l-3}} d_{l}$.

5.42. Remark : The isomorphism 5.41 is equivalent to saying that a genetic basis of the group $P=\left(D_{2^{m}}\right)^{n}$ consists of one subgroup $S$ such 
that $N_{P}(S) / S \cong \mathbf{1}$ (namely $S=P$ ), of $5^{n}-1$ subgroups $S$ such that $N_{P}(S) / S \cong C_{2}$, and, for $4 \leq l \leq m$, of $\frac{\left(3+2^{l-2}\right)^{n}-\left(3+2^{l-3}\right)^{n}}{2^{l-3}}$ subgroups $S$ such that $N_{P}(S) / S \cong D_{2^{l}}$.

Together with Assertion 3 of Corollary 5.32, this also gives the structure of genetic bases of the groups $\left(D_{2^{m}}\right)^{* n},\left(S D_{2^{m}}\right)^{* n},\left(Q_{2^{m}}\right)^{* n}$ :

5.43. Corollary : Let $P$ be one of the groups $D_{2^{m}}, S D_{2^{m}}$, or $Q_{2^{m}}$, for $m \geq 4$. Then, for any positive integer $n$, any genetic basis of the group $Q=P^{* n}$ consists :

- of one group $S$ such that $N_{P}(S) / S=\mathbf{1}$ (namely $S=Q$ ).

- of $5^{n}-1$ subgroups $S$ such that $N_{P}(S) / S \cong C_{2}$.

- for $4 \leq l \leq m-1$, of $\frac{\left(3+2^{l-2}\right)^{n}-\left(3+2^{l-3}\right)^{n}}{2^{l-3}}$ subgroups $S$ such that $N_{P}(S) / S \cong D_{2^{l}}$.

- of $2^{(n-1)(m-3)}$ subgroups $S$ such that $N_{P}(S) / S$ is isomorphic to

$$
\left\{\begin{array}{cl}
D_{2^{m}} & \text { if } P=D_{2^{m}} \\
S D_{2^{m}} & \text { if } P=S D_{2^{m}} \\
D_{2^{m}} & \text { if } P=Q_{2^{m}} \text { and } n \text { is even } \\
Q_{2^{m}} & \text { if } P=Q_{2^{m}} \text { and } n \text { is odd } .
\end{array}\right.
$$

\section{References}

[1] L. Barker. Rhetorical biset functors, rational $p$-biset functors, and their semisimplicity in characteristic zero. J. Algebra, 319(9):3810-3853, 2008.

[2] S. Bouc. Foncteurs d'ensembles munis d'une double action. J. of Algebra, 183(0238):664-736, 1996.

[3] S. Bouc. The functor of rational representations for $p$-groups. Advances in Mathematics, 186:267-306, 2004.

[4] S. Bouc. Biset functors and genetic sections for $p$-groups. Journal of Algebra, 284(1):179-202, 2005.

[5] S. Bouc. The Dade group of a p-group. Inv. Math., 164:189-231, 2006. 
[6] S. Bouc. The functor of units of Burnside rings for $p$-groups. Comm. Math. Helv., 82:583-615, 2007.

[7] S. Bouc. Rational p-biset functors. Journal of Algebra, 319:1776-1800, 2008.

[8] S. Bouc. Biset functors for finite groups, volume 1990 of Lecture Notes in Mathematics. Springer, 2010.

[9] S. Bouc and N. Mazza. The Dade group of (almost) extraspecial $p$ groups. J. Pure and Applied Algebra, 192:21-51, 2004.

[10] J. Carlson and J. Thévenaz. Torsion endo-trivial modules. Algebras and Representation Theory, 3:303-335, 2000.

[11] The GAP Group. GAP - Groups, Algorithms, and Programming, Version 4.4.12, 2008. (\protect \vrule width0pt\protect \href\{http://www.gap-system.org\}\{http://www.

[12] W. Gorenstein. Finite groups. Chelsea, 1968.

[13] I. Hambleton, L. R. Taylor, and E. B. Williams. Detection theorems for K-theory and L-theory. J. Pure and Applied Algebra, 63:247-299, 1990.

[14] P. Roquette. Realisierung von Darstellungen endlicher nilpotente Gruppen. Arch. Math., 9:224-250, 1958.

[15] T. Szamuely. Galois Groups and Fundamental Groups, volume 117 of Cambridge studies in advanced mathematics. Cambridge University Press, 2009.

Serge Bouc - CNRS-LAMFA, Université de Picardie, 33 rue St Leu, 80039, Amiens Cedex 01 - France.

email : serge.bouc@u-picardie.fr

web : http://www.lamfa.u-picardie.fr/bouc/ 